\title{
SPATIOTEMPORAL DYNAMICS OF VISUAL VERTICAL JUDGMENTS: EARLY AND LATE BRAIN MECHANISMS AS REVEALED BY HIGH-DENSITY ELECTRICAL NEUROIMAGING
}

\section{LOPEZ, ${ }^{a, b *}$ M. R. MERCIER, ${ }^{a, d, e, f}$ \\ P. HALJE ${ }^{a, g}$ AND O. BLANKE ${ }^{a, c}$}

a Laboratory of Cognitive Neuroscience, Brain Mind Institute, Ecole Polytechnique Fédérale de Lausanne (EPFL), Lausanne, Switzerland

${ }^{b}$ Institute of Psychology, University of Bern, Bern, Switzerland

${ }^{c}$ Department of Neurology, University Hospital, Geneva, Switzerland

${ }^{d}$ The Cognitive Neurophysiology Laboratory, Children's Evaluation and Rehabilitation Center, Departments of Pediatrics and Neuroscience, Albert Einstein College of Medicine, New York, USA

${ }^{e}$ The Cognitive Neurophysiology Laboratory, Program in Cognitive Neuroscience, Departments of Psychology and Biology, The City College of New York, New York, USA

${ }^{f}$ Department of Neurological Surgery, Weill Cornell Medical College, New York Presbyterian Hospital, New York, USA

${ }^{g}$ Department of Experimental Medicine, Lund University, Lund, Sweden

\begin{abstract}
Constructing and updating an internal model of verticality is fundamental for maintaining an erect posture and facilitating visuo-spatial processing. The judgment of the visual vertical (VV) has been intensively studied in psychophysical investigations and relies mainly on the integration of visual and vestibular signals, although a contribution of postural and somatosensory signals has been reported. Here we used high-density 192-channel evoked potential (EP) mapping and distributed source localization techniques to reveal the neural mechanisms of VV judgments. VV judgments (judging the orientation of visual lines with respect to the subjective vertical) were performed with and without a tilted visual frame. EP mapping revealed a sequence of neural processing steps (EP maps) of which two were specific for VV judgments. An early EP map, observed at $\sim 75-105 \mathrm{~ms}$ post-stimulus, was localized in right lateral temporo-occipital cortex. A later EP map ( $\sim 260-290 \mathrm{~ms})$ was localized in bilateral temporo-occipital and parieto-occipital cortex. These data suggest that early VV-related neural processing involves the lateral and ventral visual stream and is related to visual processing concerning orientation, attention and comparison. The later, more dorsal, activation involves multimodal cortex subtending a constantly available and updated internal model of the vertical that we can refer to for the control of

${ }^{*}$ Correspondence to: C. Lopez, Laboratory of Cognitive Neuroscience, Brain Mind Institute, Ecole Polytechnique Fédérale de Lausanne (EPFL), Swiss Federal Institute of Technology, Station 19, 1015 Lausanne, Switzerland. Tel: +41-(0)21-693-17-71; fax: +41-(0)21-693-17-70.

E-mail addresses: christophe.lopez@psy.unibe.ch or christophe.g. lopez@gmail.com (C. Lopez).

Abbreviations: ANOVA, analysis of variance; $\mathrm{CV}$, cross-validation; EEG, electroencephalography; $E P$, evoked potential; fMRI, functional magnetic resonance imaging; GEV, global explained variance; GFP, global field power; LAURA, local auto-regressive average; MT, middle temporal visual area; PET, positron emission tomography; RFT, rod and frame test; T-AAHC, topographic-atomize and agglomerate hierarchical clustering: VV, visual vertical; V1, primary visual cortex.
\end{abstract}

one's posture, actions, and visuo-spatial processing. (C) 2011 IBRO. Published by Elsevier Ltd. All rights reserved.

Key words: event-related potentials, human, subjective visual vertical, visual cortex, visual-vestibular integration.

The vertical, given by the orientation of gravity, is a fundamental spatial reference according to which human behavior on Earth has been molded. Using an internal model of the vertical, the brain can organize a proper erect posture through motor commands devoted to maintaining the body axis aligned with gravity (Pérennou et al., 2008). Such an internal model can be seen as neural processes representing the vertical, similar to internal models used to compute physical laws of motion and to estimate gravity (Angelaki et al., 2004; Merfeld et al., 1999; Snyder, 1999). Multiple observations support the view according to which the brain elaborates and uses an internal model of the vertical to achieve postural control (Barra et al., 2010) and to facilitate visual and spatial processing (Dyde et al., 2006; Indovina et al., 2005; Lopez et al., 2009).

There is behavioral evidence to suggest that the brain has developed special neural mechanisms for coding and processing vertically-oriented objects and patterns. Human spatial perception of visual stimuli is improved when these are oriented vertically or horizontally as compared to obliquely (Appelle, 1972; Orban et al., 1984). This effect has been proposed to be related to larger neural populations tuned to vertical and horizontal orientations than to oblique orientations, as shown by electrophysiological recordings in the primary visual cortex (V1) of various mammalian species, including monkeys (Mansfield, 1974), cats (Li et al., 2003), and ferrets (Coppola et al., 1998). These studies have revealed that visual patterns oriented vertically induced stronger neuronal responses than oblique patterns in V1. More recently, similar findings have been made in the middle temporal visual area (MT) of the monkey (Xu et al., 2006). Neuroimaging studies in humans, using static or moving gratings, confirmed that the human visual system is also more sensitive to vertical stimuli: gratings oriented vertically evoked stronger activity in V1, leading to larger visual evoked potentials (Maffei and Campbell, 1970) and stronger hemodynamic responses (Furmanski and Engel, 2000) than oblique gratings.

Previous neuroimaging studies used only passive presentations of oriented visual stimuli and, to the best of our knowledge, they did not investigate the brain mechanisms related to explicit judgments of the vertical, despite the fact that the subjective perception of the gravitational orienta- 
tion is crucial for sensorimotor integration (Snyder, 1999). In addition, previous studies mostly analyzed neural responses in $\mathrm{V} 1$, although many other cortical regions may contribute to the perception of the vertical (Barra et al., 2010; Kerkhoff, 1999; Pérennou et al., 2008; Yelnik et al., 2002) and to orientation discrimination (Dupont et al., 1998; Orban et al., 1997; Orban and Vogels, 1998; Taira et al., 1998; Vandenberghe et al., 1996).

To study the perception of the vertical, healthy participants are classically asked to judge the orientation of a visual line with respect to the gravitational vertical by aligning a visual target (e.g. a luminous rod) with their internal representation of the vertical (Witkin and Asch, 1948). This so-called subjective "visual vertical" (VV) usually has an accuracy of $<2$ degrees and requires mainly the integration of vestibular and visual signals, although a contribution of postural motor signals and somatosensory signals has also been reported. Vestibular cues are important for VV judgments since otolith organs sense gravitational acceleration (Bronstein, 1999; Lopez et al., 2007; Mittelstaedt, 1983; Zink et al., 1998). The VV is also influenced by postural motor signals (Bray et al., 2004; Lopez et al., 2008; Riccio et al., 1992; Van Beuzekom et al., 2001) as well as by somatosensory signals on the basis of mechanoreceptors measuring the forces acting on the joints, muscles, skin, and internal graviceptors located in the cardiovascular system, kidneys, and stomach (Barra et al., 2010; Mittelstaedt, 1983, 1992; Trousselard et al., 2004; Vaitl et al., 1997). The role of vestibular and somatosensory cues in VV judgments was confirmed in participants rotated in their frontal plane and committing $\mathrm{V} V$ judgments errors whose amplitude and direction depended on the amount of body tilt (Kaptein and Van Gisbergen, 2004; Mittelstaedt, 1983; Van Beuzekom and Van Gisbergen, 2000; Van Beuzekom et al., 2001; Vingerhoets et al., 2009). Finally, the importance of visual cues has been demonstrated by the large deviations of the VV evoked by optic flow rotating around the line of sight (Dichgans et al., 1972; Guerraz et al., 1998; Lopez et al., 2007) and oriented visual environments (Dyde et al., 2006). In the widely used and validated 'rod and frame test' (RFT) participants are required to judge the orientation of a mobile rod that is embedded in a tilted square frame (Witkin and Asch, 1948). The VV is typically deviated in the direction of the frame tilt by 2-8 degrees, depending on the participant's reliance on visual references (Bray et al., 2004; Guerraz et al., 1998; Isableu et al., 2008; Lopez et al., 2006; Luyat et al., 1997, 2005; Marendaz, 1998; Witkin and Asch, 1948; Zoccolotti et al., 1992).

Thus, what are the brain structures and mechanisms involved in VV judgments? Clinical reports indicate that several cortical regions may be involved in vertical judgments. The VV may be deviated towards or away from the lesioned side after damage to the insula, superior temporal gyrus, or posterior parietal cortex (Barra et al., 2010; Brandt et al., 1994; Darling et al., 2003; Hegemann et al., 2004; Pérennou et al., 2008; Yelnik et al., 2002). Recently, Corbett and colleagues (2009) performed an electroencephalography study in participants involved in VV judgments and demonstrated that postperceptual mechanisms (around 300 ms post-stimulus) were influenced by a tilted visual frame as well as by the orientation of the observer's body. Yet, these authors did not compare the neural correlates of VV judgments with and without visual references and did not localize the generators of the evoked potentials by the use of source localization procedures. Therefore, there is so far no published neuroimaging study in healthy participants investigating the location and timing of brain activity of $\mathrm{VV}$ judgments. This lack of empirical evidence in healthy participants is very likely a result of the imposed supine position of the commonly used neuroimaging techniques such as functional magnetic resonance imaging (fMRI) and positron emission tomography (PET). Moreover, a supine position has been shown to interfere with VV judgments (Lopez et al., 2008; Luyat et al., 1997 ) as well as with the neural mechanisms of visuospatial processing (Arzy et al., 2006), rendering neuroimaging techniques using a supine body position less indicated. Accordingly, the present neurophysiological investigation was motivated by the fact that multichannel evoked potential (EP) mapping and electrical neuroimaging allowed us to record brain signals with high temporal and spatial resolution and also allowed us to test participants in the upright position, that is, in an ecologically valid situation maintaining a corporal alignment with gravity.

Here, we used event-related potentials recorded via high-density electroencephalography (EEG), and applied topographic EP analysis and distributed source localization techniques to investigate the timing and location of brain activity during $\mathrm{VV}$ judgments and during a control task. In a forced-choice orientation-recognition design healthy participants judged how visual line segments were oriented with respect to the gravitational vertical when only lines were presented on the visual background. Because the perception of $\mathrm{V} V$ has been shown to depend on external signals such as the orientation of the visual environment, we also used a computer-adaptation of the RFT (with a square tilted by $20^{\circ}$ clockwise) in order to investigate how visual references can change the underlying neural mechanisms of $\mathrm{VV}$ judgments.

\section{EXPERIMENTAL PROCEDURES}

\section{Participants}

Twelve healthy volunteers (six females and six males, mean \pm standard deviation $25.8 \pm 4$ years) participated in this experiment. All of them were right-handed, as confirmed by the Edinburgh Handedness inventory (Oldfield, 1971). They had normal or corrected-to-normal vision and declared no history of vestibular, neurological, or psychiatric disease. Experimental procedures were approved by the local Ethics Committee (University Hospital of Lausanne) and followed the ethical recommendations laid down in the Declaration of Helsinki. All participants gave written informed consent after they were fully informed about the study. 


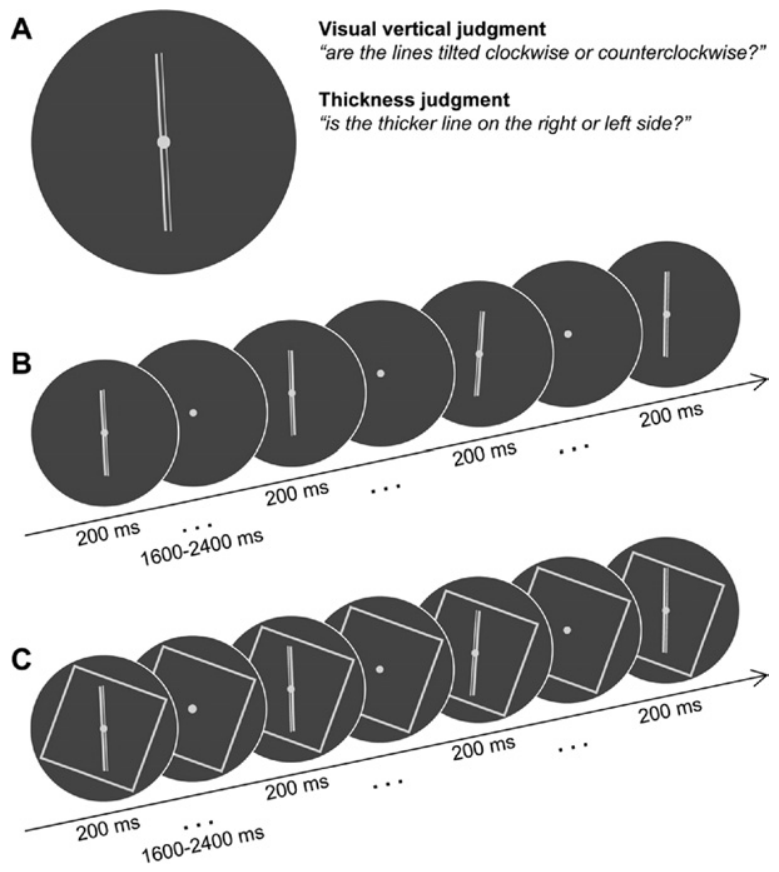

Fig. 1. Visual stimuli used during visual vertical and thickness judgments $(A)$. Two parallel lines (a thick line and a thin line) were presented at the center of the screen, slightly tilted clockwise or counterclockwise with respect to the gravitational vertical (from $0.5^{\circ}$ to $4^{\circ}$ ). The thicker line was located either to the right or to the left side of the screen. These lines were presented for $200 \mathrm{~ms}$ without references in the background $(B)$ or surrounded by a square tilted by $20^{\circ}$ in the clockwise direction (C). Between two successive presentations of visual stimuli, a fixation point alone was presented $(B)$, or a fixation point and a tilted square were presented (C), and the interstimulus intervals ranged from 1600 to $2400 \mathrm{~ms}$.

\section{Visual stimuli}

Visual stimuli were presented in a dark and soundproof room on a 19 inch high resolution Cathode Ray Tube computer screen (ViewSonic, graphic series G90f+) with a refresh rate of $100 \mathrm{~Hz}$. The screen was the only source of light in the room and was located $1 \mathrm{~m}$ from the participant's eyes. The screen was covered with a black circular frame to narrow the visual scene to a circular area ( $25 \mathrm{~cm}$ in diameter, subtending $\sim 14^{\circ}$ of the visual field) and to exclude any vertical and horizontal references (see Lopez et al., 2009 for similar procedures).

Stimuli were presented as $1182 \times 1024$ pixel images using the E-Prime 1.1 software (E-Studio, Psychology Software Tools, Pittsburgh, PA, USA). The stimuli comprised two parallel line segments (light gray, $12.4 \mathrm{~cm}$ long, $\sim 7^{\circ}$ of visual angle) presented on a dark gray background (Fig. $1 \mathrm{~A})$. One of the two lines was thicker ( $2 \mathrm{~mm}$ in width, $\sim 0.23^{\circ}$ of the visual field) than the other line $(1 \mathrm{~mm}$ in width, $\sim 0.11^{\circ}$ of the visual field). A fixation point $(6 \mathrm{~mm}$ in diameter) was located at the centre of the parallel lines and was vertically and horizontally centered within the visual display. The parallel line segments were presented tilted either counterclockwise or clockwise with angles of $0.5^{\circ}, 1^{\circ}, 1.5^{\circ}, 2^{\circ}, 2.5^{\circ}, 3^{\circ}, 3.5^{\circ}$, and $4^{\circ}$ with respect to the gravitational vertical. No vertical lines were presented because previous neuroimaging studies demonstrated that vertical lines evoke different and stronger brain activity than tilted lines (Maffei and Campbell, 1970; Furmanski and Engel, 2000). Finally, the position of the thick line (leftmost or rightmost) was balanced across trials for each orientation of the line segments.
The oriented line segments were presented in two visual environments:

(a) Without visual references: The lines were presented on the gray background without any visual reference (Fig. 1B).

(b) With tilted visual references: A computer adaptation of the classical RFT (Witkin and Asch, 1948) was used in order to investigate the effects of visual references on the mechanisms of VV judgments (Fig. 1C). The same two line segments as in (a) were presented surrounded by a square frame (light gray, $15.7 \times 15.7 \mathrm{~cm}$, subtending $9^{\circ}$ of the visual field), which was tilted by $20^{\circ}$ in the clockwise direction. This amplitude of the frame tilt has been shown to evoke large deviations of the $\mathrm{VV}$ toward the frame tilt (Lopez et al., 2006; Marendaz, 1998; Zoccolotti et al., 1992).

\section{Procedures}

Experiments were conducted with participants seated on a chair, straight and motionless. They were asked to fixate the fixation point. Participants were required to perform two visuo-spatial tasks with identical visual stimuli and an identical response mode (manual response). The instructions to the participant differed depending on the task (Fig. 1A):

(a) VV judgment task: In a forced-choice orientation-recognition task, participants were asked to indicate if the line segments were tilted clockwise or counterclockwise with respect to the vertical.

(b) Thickness judgment task: As a control task, participants were asked to indicate if the thicker line segment was on the right or left, irrespective of line orientation. This control task was used in order to have participants involved in a visuo-spatial task similar to the VV judgment task in terms of physical properties of the visual stimuli (luminance, contrast, color, form, and size), motor responses (key presses with the right index and middle fingers), and difficulty.

The overall experiment was composed of four separate blocks (two tasks $\times$ two visual environments) and the order of the blocks was randomized across participants. Each block consisted of 256 stimuli (eight angles $\times$ two tilt directions $\times$ two thickest line locations $\times$ eight repetitions) with the line segments presented for $200 \mathrm{~ms}$ in random order. A fixation point was continuously presented at the center of the screen, during each line presentation and between stimulus presentations. Note that for the RFT, the tilted square was permanently shown; only the line segments were presented for $200 \mathrm{~ms}$, as in the condition without visual reference. The mean interstimulus interval was $2000 \mathrm{~ms}$ and ranged from $1600-2400 \mathrm{~ms}$.

Responses were given using a response box (Psychology Software Tools, Pittsburgh, PA, USA) with the right hand. Participants were instructed to press a button with the index finger if the line segments were tilted counterclockwise (VV judgments) or if the thicker line was located on the left (thickness judgments). Conversely, they had to press a button with the middle finger if the line segments were tilted clockwise (VV judgments), or if the thickest line was located on the right (thickness judgments). In both tasks, they were instructed to answer as fast and accurately as possible. Prior to the EEG recording, all participants completed one practice block of each task (64 trials per block) for familiarization with the response box and the experimental procedures.

\section{Behavioral data recording and analysis}

For each trial, the measured dependent variables were the participant's answers (clockwise vs. counterclockwise in the VV judgment tasks; left vs. right in the thickness judgment tasks) and reaction times (in $\mathrm{ms}$ ). For the $\mathrm{VV}$ judgment tasks, we calculated the percentage of responses indicating a clockwise tilt of the lines. The percentage of clockwise answers was plotted as a function of the line orientation and we fitted the data with a 
sigmoid psychometric function using least-squares regression (Matlab 7.6, MathWorks Inc., Natick, MA, USA). The regression was performed on the individual participant data with the initial condition $b_{1}=2, b_{2}=0$. The non-linear regression was performed using the nlinfit function that computes fits using the LevenbergMarquardt algorithm (Seber and Wild, 2003). The confidence intervals of the estimated parameters were estimated with the nlparci function. The equation of the sigmoid function was:

$$
f(x)=\frac{1}{1+e^{-b_{1} x+b_{2}}}
$$

where $x$ was the line orientation, and $b_{1}$ and $b_{2}$ were parameters determined by the regression. The regression was performed on the individual participant data. From the sigmoid parameters we could extract the line orientation corresponding to $50 \%$ of clockwise answers (see Lopez et al., 2009 for a similar approach), since:

$$
0.5=\frac{1}{1+e^{-b_{1} x+b_{2}}} \Leftrightarrow x=\frac{b_{2}}{b_{1}} .
$$

The $50 \%$ point obtained is expected to be a relevant evaluation of the so-called "subjective VV" (see Dyde et al., 2006 for a similar evaluation method of the subjective VV, and Foxe et al., 2003 for an evaluation of the perceived line midpoint). Finally, for the thickness judgment tasks, the percentage of correct answers and the mean reaction time for correct answers were calculated.

The percentage of clockwise answers (VV judgments) and the percentage of correct answers (thickness judgments) were analyzed using repeated-measures analyses of variance (ANOVA) with the Line orientation and the Visual references as within-subjects factors. The subjective $\mathrm{V}$ was also analyzed using repeated-measures ANOVA to investigate the effect of the references in the visual surrounding. To compare task difficulty, reaction times were analyzed using repeated-measures ANOVA with Task, Line orientation, and Visual reference as within-subjects factors. Results were considered statistically significant for $P<0.05$.

\section{EEG data acquisition and analysis}

Continuous EEG was recorded with a Biosemi Active system (Biosemi Inc., Amsterdam, the Netherlands) from 192 active scalp electrodes in a Faraday cage ( $2048 \mathrm{~Hz}$ sampling rate). The CMS-DRL reference electrodes were located close to the apex. Eye movements were monitored using four additional electrodes, two positioned vertically above and below the non-dominant eye, and two electrodes positioned horizontally near the left and right outer canthi.

We calculated stimulus-locked EPs for each condition (two Tasks $\times$ two Visual references) using the average reference (Lehmann and Skrandies, 1980; Murray et al., 2008a). Epoch onset was set to $200 \mathrm{~ms}$ before stimulus onset and epoch duration was $500 \mathrm{~ms}$. Data were band-pass filtered $(1-30 \mathrm{~Hz})$ and corrected with respect to the baseline, which was defined as the $100 \mathrm{~ms}$ pre-stimulus period. Data were visually inspected to reject any epoch with eye blinks, eye movements, and any source of transient noise using the Cartool 3.33 software (by D. Brunet, Geneva, Switzerland; http://brainmapping.unige.ch/Cartool.htm). Trials yielding incorrect responses (for the thickness judgment tasks) or no response were excluded from the EPs computation. The percentage of trials discarded for incorrect answers averaged (mean \pm SD) $4.9 \pm 6.4 \%$ for the thickness judgments without frame and $5.6 \pm 6.9 \%$ for the thickness judgments with frame. The percentage of trials with no response averaged $1.1 \pm 1.5 \%$ for the $\mathrm{VV}$ judgments without frame, $0.7 \pm 1.0 \%$ for the $\mathrm{VV}$ judgments with frame, $1.0 \pm 1.7 \%$ for the thickness judgments without frame, and $0.9 \pm 1.5 \%$ for the thickness judgments with frame. For each participant, artifacted electrodes in the EPs were interpolated using a
3D spline interpolation (Perrin et al., 1987). Before group averaging, EPs were temporally realigned such as the peak of the P1 component was at $100 \mathrm{~ms}$ post-stimulus for each participant and each experimental condition (Morand et al., 2000). Group averaging was calculated across participants by averaging individual EPs normalized to their "global field power" (GFP). The GFP is a reference-independent measure of the response strength and it can be computed as the standard deviation of all scalp electrodes at a given time point $t$ (Lehmann and Skrandies, 1980). For each experimental condition, the instantaneous GFP can be defined as:

$$
\mathrm{GFP}_{u}=\sqrt{\frac{\sum_{i=1}^{n} u_{i}^{2}}{n}}
$$

where $n$ is the number of electrodes used in the montage, and $u_{i}$ is the average-referenced potential of the $t^{\text {th }}$ electrode (Murray et al., 2008a).

Modulation of the strength of the electrical field. The modulation of the strength of the electrical field on the scalp across conditions was analyzed using the instantaneous GFP for each participant. The GFP was statistically analyzed with point-by-point paired $t$-tests whose results were corrected for temporal autocorrelation by using the constraints of 11 consecutive data points reaching the 0.01 level of significance (for similar procedures, see Murray et al., 2004, 2008b; Shpaner et al., 2009).

Topographic pattern analysis. EPs were analyzed on the basis of the spatial variations of the distribution of the scalp voltage over time and between experimental conditions, an approach known in the literature as "topographic EP analysis" and used in many previous EP studies by others and us (Arzy et al., 2006; Blanke et al., 2005; Lehmann et al., 1987; Mercier et al., 2009; Michel et al., 2001, 2004; Morand et al., 2000; Murray et al., 2004, 2008b; PascualMarqui et al., 1995; Thierry et al., 2006; Thirioux et al., 2010; for a recent review see Murray et al., 2008a). This approach defines stable map topographies along time that represent "functional microstates of brain activity". The general idea is that scalp topographies do not fluctuate randomly but are characterized by successive periods of stability, which we refer to here as EP maps. The duration of these EP maps usually range from 10 to $100 \mathrm{~ms}$ depending on the perceptive or cognitive processes involved (Arzy et al., 2006; Blanke et al., 2005; Mercier et al., 2009; Morand et al., 2000; Rauss et al., 2009; Thierry et al., 2006; Thirioux et al., 2010). These EP maps were identified from the grand-averaged EPs across the four experimental conditions using the T-AAHC hierarchical clustering algorithm (for "Topographic-Atomize and Agglomerate Hierarchical Clustering", Cartool 3.33, Geneva, Switzerland; for a detailed description see Murray et al., 2008a). These EP maps are the mean maps over the period where the segment was found across conditions. The optimal number of EP maps describing the group-averaged EPs was determined by using a cross-validation (CV) criterion optimizing the degrees of freedom and the explained variance (Pascual-Marqui et al., 1995; Murray et al., 2008a). This CV criterion is a modified version of the predictive residual variance and can be defined as:

$$
C V=\hat{\sigma}_{\mu}^{2} \cdot\left(\frac{n-1}{n-1-q}\right)^{2}
$$

with

$$
\hat{\sigma}_{\mu}^{2}=\frac{\sum_{t=1}^{t_{\max }}\left(\|u(t)\|^{2}-\left(T_{t} \cdot u(t)\right)^{2}\right)}{t_{\max } \cdot(n-1)}
$$

where $q$ is the number of EP maps, $n$ is the number of electrodes used in the montage, $T_{t} u(t)$ is the scalar product between the 
template maps $T_{t}$ and the data $u(t)$. The value of $q$ minimizing the CV criterion indicated the optimal number of EP maps.

In a second step, the dominant EP maps identified in the group-averaged data were fitted to the EPs of each participant by using spatial fitting procedures (Murray et al., 2008a). For each time point of the participant's EP, the scalp topography was compared to each EP map by using normalized spatial correlation, and it was labeled according to the one with which it correlated best. From this fitting procedure, we determined two parameters representing the occurrence of a given EP map for each experimental condition: the duration of the map (number of time-points that were assigned to the EP map) and the global explained variance (GEV). The GEV described how well an EP map fits during a certain time period and can be computed as the sum of the explained variances (ev) weighted by the GFP at each time point $t$ :

$$
\mathrm{GEV}=\frac{\sum_{t=1}^{t_{\max }}\left(\mathrm{GFP}^{2} \times \mathrm{ev}\right)}{\sum_{t=1}^{t_{\max }} \mathrm{GFP}^{2}}
$$

The GEV and duration (in ms) of the dominant EP maps were analyzed using repeated-measures ANOVA with the Task (VV judgment task, thickness judgment task) and Visual reference (without visual references, with tilted visual references) as withinsubjects factors.

Source localization. The neural generators for the EP map related to VV judgments were estimated using a distributed linear inverse solution based on a Local Auto-Regressive Average (LAURA) model as used previously (Gonzalez Andino et al., 2001; Grave de Peralta Menendez et al., 2004). Map topographies were downsampled to a 111-channel montage because the LAURA algorithm we used has been tested extensively for this configuration (Mercier et al., 2009; Schwabe et al., 2009). The solution space was calculated on a realistic head model that included 4024 nodes equally distributed within the gray matter of the Montreal Neurological Institute's average brain. Finally, coordinates of the neural generators were reported in Talairach coordinates (Talairach and Tournoux, 1988) and the labeling of regions was performed using the Talairach software (by J. Lancaster and $\mathrm{P}$. Fox, University of Texas, Health Science Center San Antonio; http://www.talairach.org/).

Statistical analysis at the source level was performed at the level of each inverse solution point. For each participant and each experimental condition, we determined the mean corresponding EP map during the time-windows for which topographical pattern analysis revealed significantly different EP maps (VV judgments: 75-105 ms; thickness judgments: 260-290 ms). LAURA was applied to the individual EP maps for each experimental condition and inverse solutions were subjected to statistical analysis consisting of paired $t$-test for each solution point (see Mercier et al., 2009; Rauss et al., 2009).

\section{RESULTS}

\section{Behavioral data}

Behavioral data collected during the $\mathrm{V}$ judgment tasks are presented in Fig. 2A. A repeated-measures ANOVA conducted on the percentage of clockwise answers revealed a significant main effect of Line orientation $\left[F_{(15,150)}=91.19\right.$; $P<0.001]$ and a nearly significant interaction of Line orientation $\times$ Visual reference $\left[F_{(15,150)}=1.70 ; P=0.05\right]$. A repeated-measures ANOVA ran on the subjective $\mathrm{V}$ extracted from the individual sigmoid curves indicated that the refer- ences in the visual surrounding significantly influenced the $\mathrm{V}$ judgment [main effect of Visual reference: $F_{(1,11)}=6.33$; $P<0.05$ ] (Fig. 2B). Thus, as predicted, in the RFT the subjective $\mathrm{V}$ was significantly deviated towards the tilted frame (clockwise) as compared to the condition without frame.

For the control tasks (thickness judgment) there was no main effect of Line orientation on the percentage of correct answers $\left[F_{(15,165)}=1.09 ; P=0.37\right]$. Performance was also the same with and without the tilted frame $\left[F_{(1,11)}=0.73 ; P=0.41\right]$ (Fig. $2 \mathrm{C}$ ). As predicted, the thickness judgment was not influenced by the line orientation and the visual reference.

Regarding reaction times (Fig. 2D), there was no difference between experimental and control tasks (mean \pm SEM, VV judgment: $512 \pm 98$ ms; thickness judgment: $\left.510 \pm 100 \mathrm{~ms} ; F_{(1,11)}=0.02 ; P=0.92\right)$. Reaction times were also not affected by the Visual reference $\left[F_{(1,11)}=0.79 ; P=0.39\right]$ in the $\mathrm{VV}$ judgment tasks or the thickness judgment tasks, suggesting uniform task difficulty across conditions.

\section{Modulation of the strength of the electrical field}

The VV and thickness judgments evoked clear stimuluslocked EP. Fig. 3 illustrates group-averaged EPs at the level of four posterior scalp electrodes where the largest waveforms were recorded. The modulation of the global strength of the electrical field on the scalp was analyzed by calculating for each participant and each condition the global field power (GFP), which can be taken as the spatial standard deviation of all scalp electrodes (Fig. 4). Results indicated that the GFP was significantly different between $\mathrm{V}$ judgments and thickness judgments without visual reference (Fig. 4A), around a time period corresponding to the EP Map ${ }_{V v 75}$ identified by the segmentation procedure (see below "Topographic EP analysis"). Such difference was not observed between $\mathrm{V} V$ judgments and thickness judgments with visual reference (Fig. 4B). Finally, the comparison of the GFP during $\mathrm{VV}$ judgments performed with and without visual reference, thus revealing a frame effect, was observed for a sustained time period starting around $260 \mathrm{~ms}$, and overlapping with the time period during which EP Map ${ }_{v \text { 260 }}$ was shown by the segmentation procedure (Fig. 4C).

\section{Topographic EP analysis}

The first step of the topographic EP analysis consists of applying a clustering algorithm to the group-averaged EPs across all conditions (VV and thickness judgments with and without visual references) in order to identify clusters of stable voltage topography. This analysis was performed on the group-averaged EPs and revealed two different time segments of stable voltage topography (EP maps) during the 75-105 ms period: one for the VV judgments $\left(M p_{V V 75}\right)$ and one for the thickness judgment Map $_{\mathrm{T} 75}$; Fig. 5A, B). In addition, two other functional microstates during the 260-290 ms period were found to be representative for the $\mathrm{VV}$ judgments $\left(\mathrm{Map}_{\mathrm{V} V 260}\right)$ and for the thickness judgments $\left(\mathrm{Map}_{\mathrm{T} 260}\right)$. The spatial configuration of these EP maps is illustrated in Fig. 5C. All other EP maps 

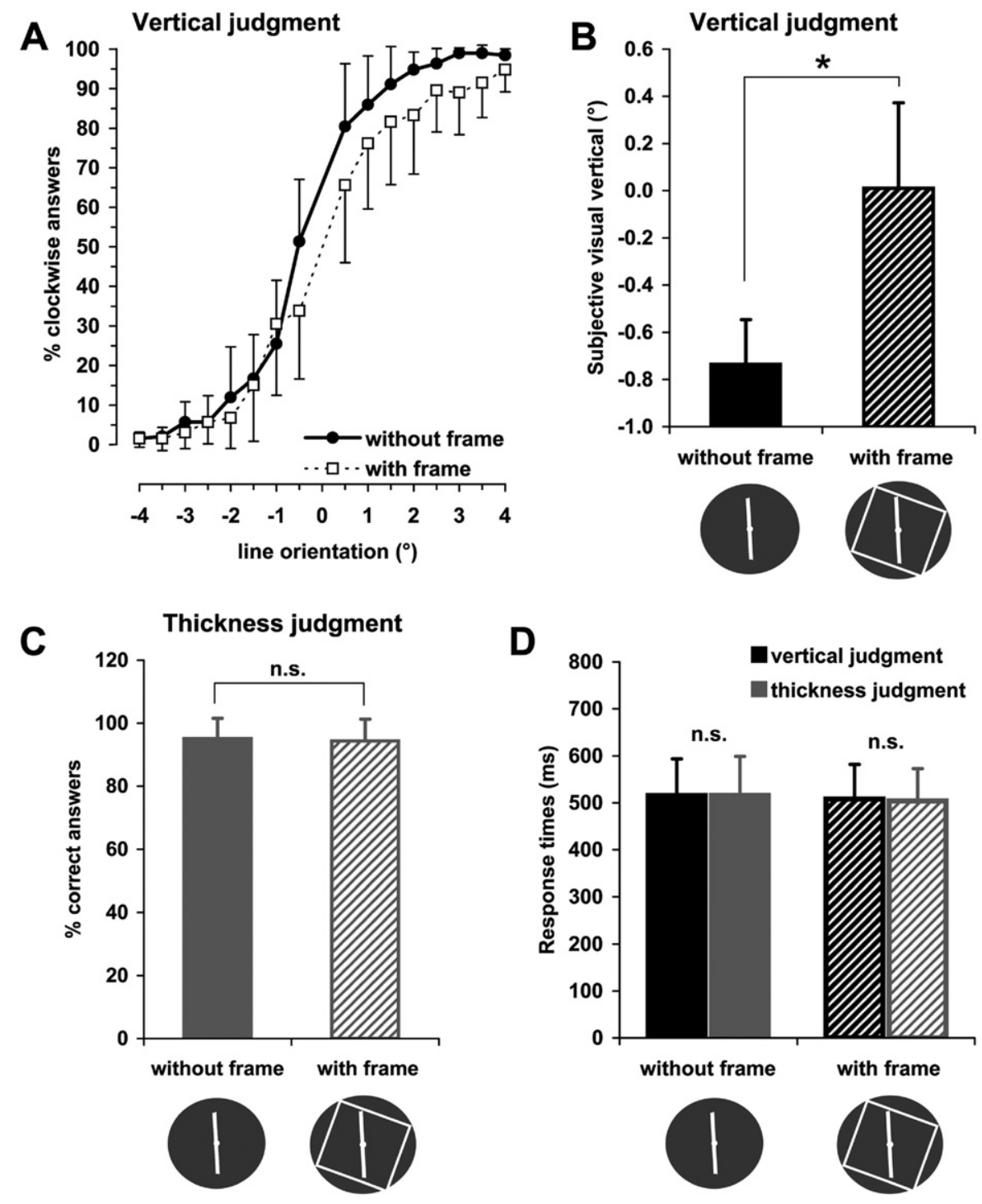

Fig. 2. Behavioral data for the visual vertical and thickness judgments. (A) Mean percentage of visual lines perceived as tilted clockwise is shown as a function of the line orientation and visual references (without references, black symbols vs. with a square frame tilted by $20^{\circ}$ clockwise, open symbols). Line orientation refers to the amplitude of the lines deviation with respect to the gravitational vertical (positive values refer to clockwise deviations). (B) The histograms represent the mean subjective visual vertical (in degrees with respect to the gravitational vertical, positive values refer to clockwise deviations) calculated from the individual psychometric functions (without references, filled histogram; with the tilted frame, dashed histogram). Vertical bars represent the standard error of the mean (SEM). There was a significant influence of the visual references, deviating the perceived vertical clockwise $\left({ }^{*} P<0.05\right)$. (C) The histograms represent the mean $( \pm S E M)$ percentage of correct answers in the thickness judgment tasks. There was no significant influence of the visual references. (D) Mean ( \pm SEM) reaction times showing homogeneous task difficulty across tasks (vertical vs. thickness judgments) and visual reference (without vs. with the tilted frame).

before, between or after these periods were similar between both tasks. No EP map was found to reflect the influence of the visual references.

Brain activity at 75-105 ms. Cluster analysis of the group-averaged EPs revealed that Map $\mathrm{vv75}_{\mathrm{V}}$ was observed between $\sim 75$ and $105 \mathrm{~ms}$ after visual stimulus onset, and Map $_{\mathrm{T} 75}$ was present between $\sim 78$ and 108 ms post-stimulus (Fig. 5A, B). In a second step of our topographic data analysis we assessed whether results from the clustering analysis were confirmed by statistical analysis by using a "fitting procedure" which is based on the spatial correlation between the template EP maps resulting from the groupaveraged EPs and the EPs of each single participant separately (see Experimental procedures). The fitting procedure revealed that $M_{\mathrm{av} 75}$ is more representative of the $\mathrm{VV}$ judgments and that $\mathrm{Map}_{\mathrm{T} 75}$ is more representative of the thickness judgments at the level of the individual EPs (Fig. 5D). Repeated-measures ANOVAs indicated that Map $_{v \text { 75 }}$ was significantly more often present during the VV judgments than the thickness judgments in the individual EPs [main effect of Task: $F_{(1,11)}=4.94 ; P<0.05$ ]. The GEV of $M_{\text {T75 }}$ was also significantly higher for the VV 

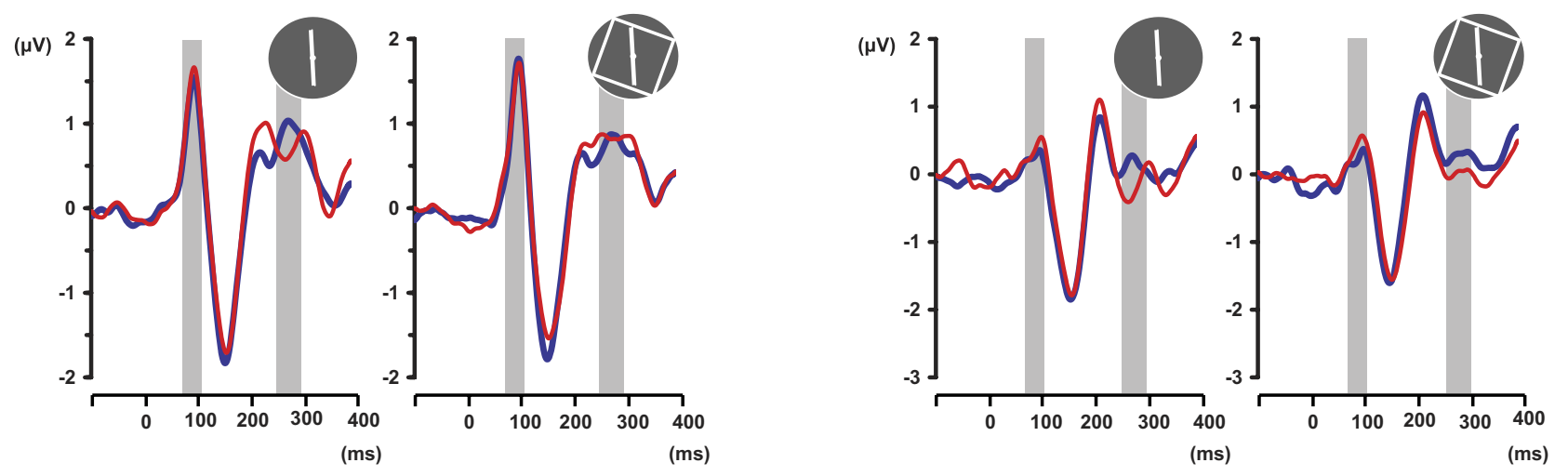

vertical judgment thickness judgment
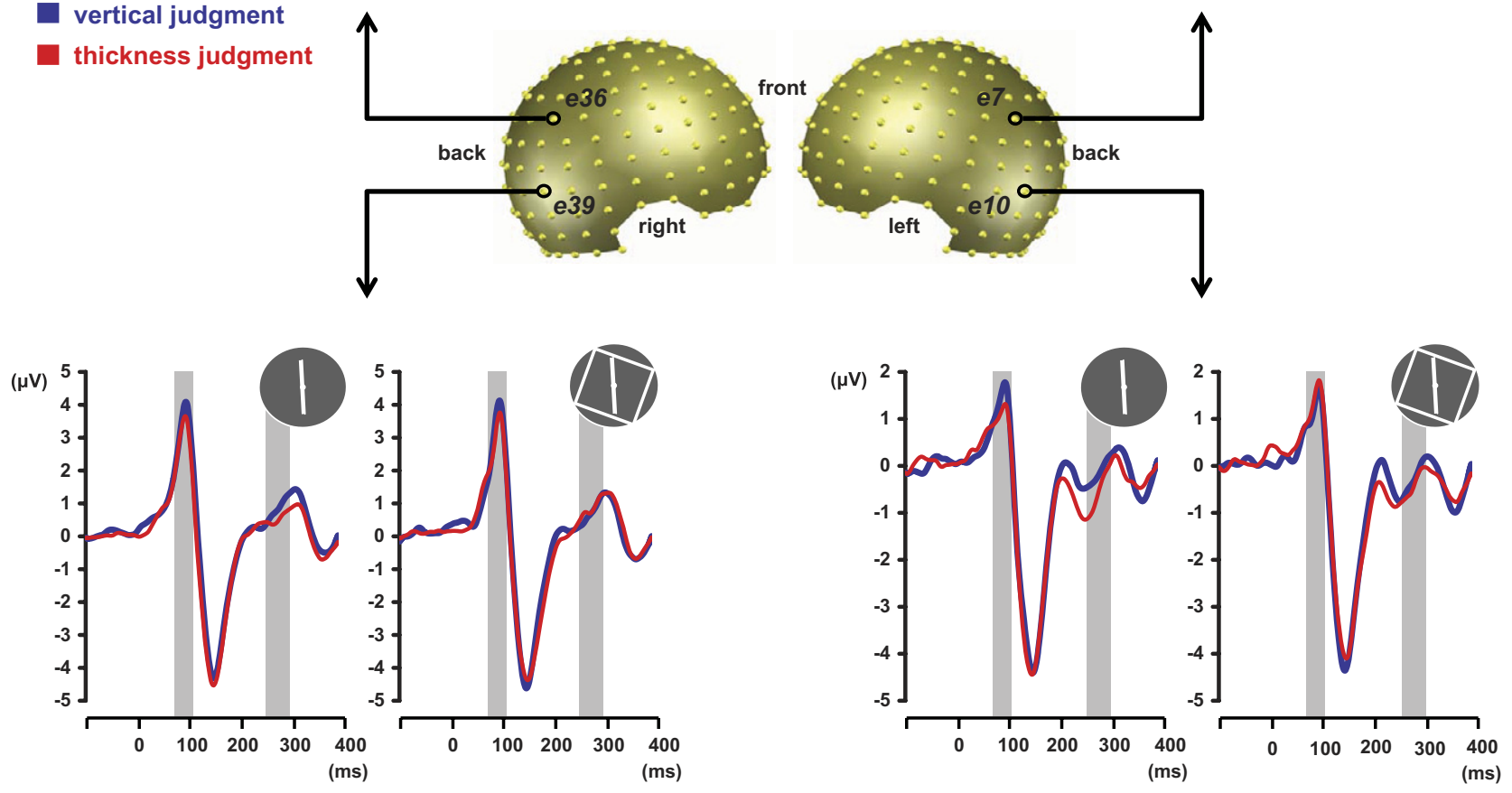

Fig. 3. Group-averaged ERPs calculated for the VV judgments and the thickness judgments for two temporo-occipital (e39, e10) and two parieto-occipital (e36, e7) electrodes. Data are shown separately for the judgments performed with and without the tilted frame from $100 \mathrm{~ms}$ pre-stimulus to $400 \mathrm{~ms}$ post-stimulus. Grey areas represent the period during which different EP maps have been identified by the segmentation procedure. For interpretation of the references to color in this figure legend, the reader is referred to the Web version of this article.

judgments than for the thickness judgments in the individual EPs [main effect of Task: $F_{(1,11)}=5.18 ; P<0.05$ ]. No significant effect of Visual reference was found for the duration $\left[F_{(1,11)}=0.28 ; P=0.61\right]$ and GEV $\left[F_{(1,11)}=0.02\right.$; $P=0.88$ ] of $M a p_{v 755}$, and no significant interaction of Task $\times$ Visual reference was found for these variables (all $F<2.02$ and $P>0.18$ ). By contrast, Map ${ }_{T 75}$ lasted significantly longer in the thickness judgments than in the $\mathrm{VV}$ judgments in the individual EPs [main effect of Task: $F_{(1,11)}=4.94 ; P<0.05 ;$ Fig. 5D]. There was no influence of Visual reference on the duration of $\operatorname{Map}_{\mathrm{T} 75}\left[F_{(1,11)}=0.14\right.$; $P=0.72]$. In a second "fitting" procedure aimed at refining the measure of the presence of Map $\mathrm{vv75}_{\mathrm{V}}$ when performing $\mathrm{VV}$ judgments, and the presence of $\mathrm{Map}_{\mathrm{T} 75}$ when performing thickness judgments, Map ${ }_{V 775}$ and $M_{175}$ were opposed (competitive fitting) to the adjacent EP maps preceding and following them (Fig. 5E). A repeated-measures ANOVA revealed a significant interaction of Task $\times$ Visual reference $\left[F_{(1,11)}=4.98 ; P<0.05\right]$. Whereas Map $\mathrm{vv75}_{\mathrm{V} 5}$ was more present during $\mathrm{V} V$ judgments without visual reference than with visual reference $(P<0.05)$, the duration of Map $_{\mathrm{T} 75}$ did not differ between the condition with and without visual reference $(P=0.7)$.

Brain activity at 260-290 ms. Cluster analysis of the group-averaged EPs revealed the presence of Map $\mathrm{VV}_{\mathrm{V} 260}$ between $\sim 260$ and $290 \mathrm{~ms}$ after visual stimulus onset and of $\mathrm{Map}_{\mathrm{T} 260}$ between $\sim 255$ and 285 ms after stimulus onset (Fig. 5A, B). We applied the same statistical analysis as done for the earlier EP period. A first fitting procedure opposing Map $\mathrm{Vv}_{\mathrm{V} 260}$ and $\mathrm{Map}_{\mathrm{T} 260}$ in the individual EPS recorded during the $\mathrm{VV}$ and thickness judgments (Fig. 5F) confirmed that Map ${ }_{\mathrm{VV} 260}$ was significantly more present during the $\mathrm{VV}$ judgments than during the thickness judgments [main effect of Task: $F_{(1,11)}=6.75 ; P<0.05$ ]. The GEV of Map ${ }_{\mathrm{V} V 260}$ was also significantly higher for the VV 
A

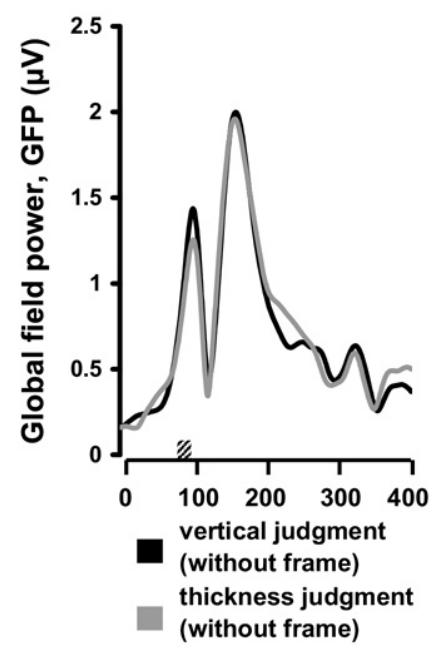

B

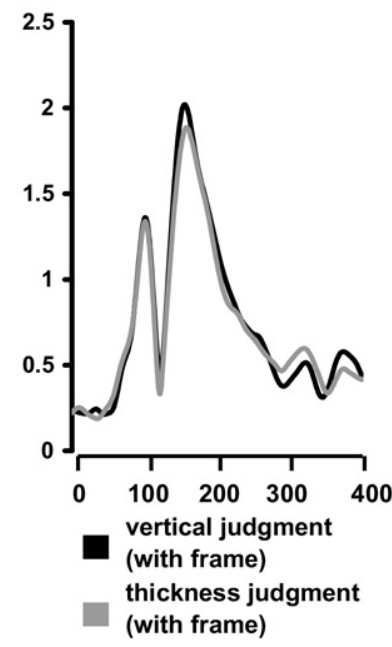

C

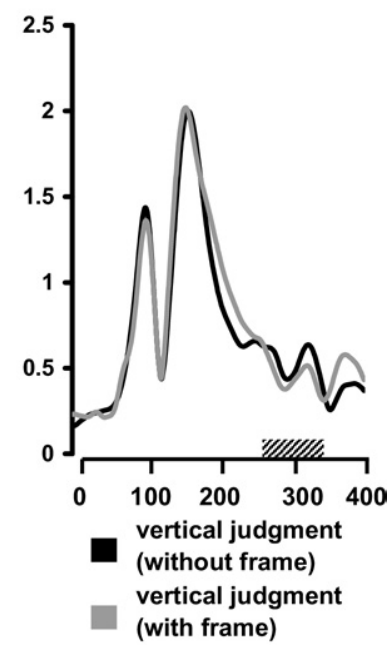

Fig. 4. Comparisons of the group-averaged global field power (GFP) calculated for the VV and the thickness judgment tasks performed without visual reference $(A)$ and with a tilted frame $(B)$. (C) Effect of the visual reference on the mean GFP during VV judgments. Hatched areas on the $x$-axis represent the periods during which the two conditions differed significantly $(P<0.01$; paired $t$-test with temporal correction).

judgments as compared to the control task [main effect of Task: $\left.F_{(1,11)}=6.05 ; P<0.05\right]$. No significant effect of Visual reference was found for the duration $\left[F_{(1,11)}=0.14\right.$; $P=0.72]$ and GEV $\left[F_{(1,11)}=1.90 ; P=0.20\right]$ of Map ${ }_{\mathrm{Vv} 260}$, and no significant interaction of Task $\times$ Visual reference was found for both of these variables (all $F<1.62$ and

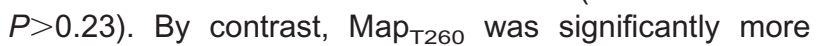
present during the thickness judgments than during the $\mathrm{VV}$ judgments in the individual EPs [main effect of Task: $F_{(1,11)}=6.75 ; P<0.05$; Fig. 5F]. Again, there was no influence of Visual reference on the duration of $\mathrm{Map}_{\mathrm{T} 260}$ $\left[F_{(1,11)}=0.14 ; P=0.72\right]$. In a second "fitting" procedure, Map $_{\mathrm{V} \text { 260 }}$ and Map $_{\mathrm{T} 260}$ were opposed to the adjacent EP maps preceding and following them, but no influence of Visual reference was found.

\section{Source localization}

For the early EP maps $\left(\mathrm{Map}_{\mathrm{VV75}}\right.$ and $\mathrm{Map}_{\mathrm{T} 75}$ ), both VV and thickness judgments involved generators within the right lateral temporo-occipital cortex (Fig. 6A, B). For $M_{\mathrm{V}} \mathrm{p}_{\mathrm{V} 75}$, the maximal peak of activation (Talairach coordinates $x, y, z=+41,-70,-4)$ was in the right inferior occipital gyrus (Brodmann area 18), extending to the middle occipital gyri (area 19). To get a better estimate of the difference between source estimations of activities related to $\mathrm{VV}$ and thickness judgments, we calculated the groupaveraged difference between source estimation for the VV and thickness judgments (for similar procedures see Murray et al., 2008b; Spierer et al., 2007; Thirioux et al., 2010). Differences between source estimation involved the temporo-occipital cortex bilaterally (Fig. 6C). The maximal difference was located in the right inferior occipital gyrus $(x, y, z=+35,-81,-4)$.

For the late EP maps $\left(\operatorname{Map}_{\mathrm{V} 260}\right.$ and $\left.\mathrm{Map}_{\mathrm{T} 260}\right)$, both $\mathrm{VV}$ and thickness judgments involved the temporo-occipital and parieto-occipital cortex bilaterally (Fig. 6D, E).
Map $_{\mathrm{Vv} 260}$ was localized to the left and right temporo-occipital cortex (extending dorsally) and to left parieto-occipital cortex. The maximal peak of activation was in the left temporo-occipital cortex $(x, y, z=-47,-58,0)$, extending to the left middle occipital gyrus and inferior temporal gyrus (area 19). A separate activation was found in left parieto-occipital cortex $(x, y, z=-23,-85,+33)$. The maximal peak of activation in the right hemisphere was located in the temporo-occipital cortex $(x, y, z=+47,-58,0)$ extending dorsally to the inferior temporal gyrus. An additional source was found in the right middle and inferior temporal gyrus (area $21 ; \mathrm{x}, \mathrm{y}, \mathrm{z}=+53,-12,-17$ ). Differences between source estimation involved the temporo-occipital cortex bilaterally, as well as the posterior parietal and inferior frontal cortex in the right hemisphere (Fig. $6 F)$. The maximal difference was found in the left inferior temporal gyrus $(x, y, z=-41,-69,0)$.

\section{Statistics on inverse solutions}

Results from the statistical analyses at the level of the inverse solutions revealed several brain regions at a $P$ value of 0.01 (uncorrected values). During the time-window 75-105 ms, differential activation between VV judgments and thickness judgments revealed the left insula (Fig. 7A). The same comparisons, but during the later activation (time-window 260-290 ms), revealed differences in the left parieto-occipital and left temporal cortex (Fig. 7B).

\section{DISCUSSION}

The aim of this study was to investigate the brain mechanisms involved in the perception of the VV. Using high-density 192-channel EEG it was possible to record brain activity and performance in $\mathrm{V}$ judgments in an ecologically valid situation (upright body axis aligned with gravity) whereas 

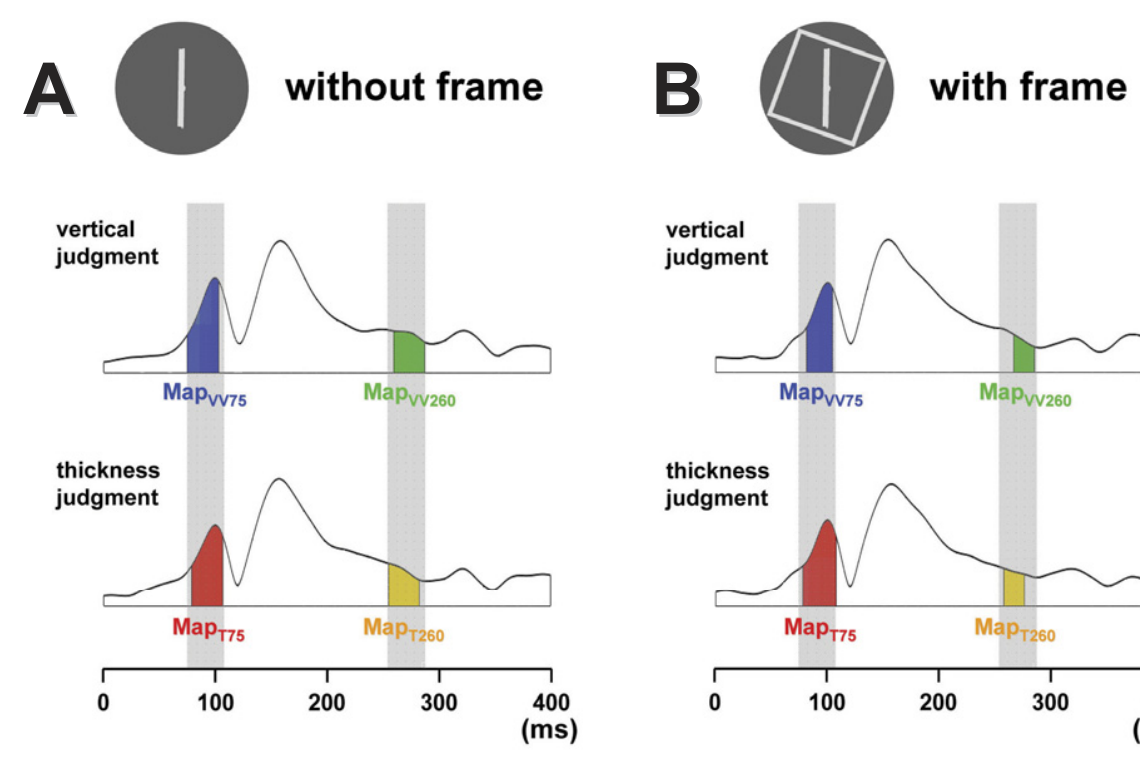

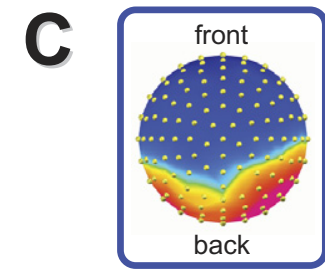

Map $_{\text {vv75 }}$

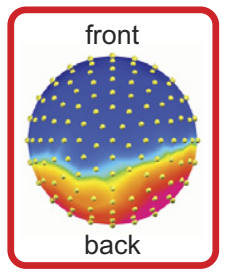

Map $_{\mathrm{T} 75}$

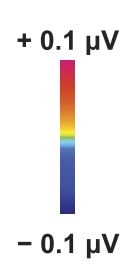

$-0.1 \mu \mathrm{V}$

without frame E $\mathrm{G}$ U with frame

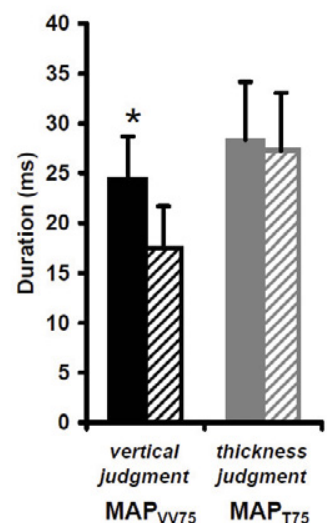

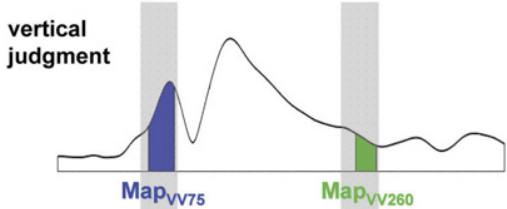

thickness judgment

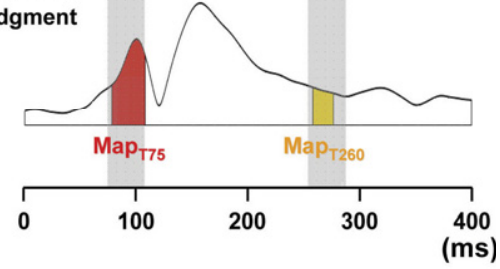

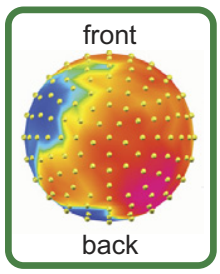

Map $_{\text {vv260 }}$

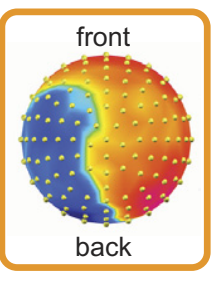

Map $_{\text {T260 }}$
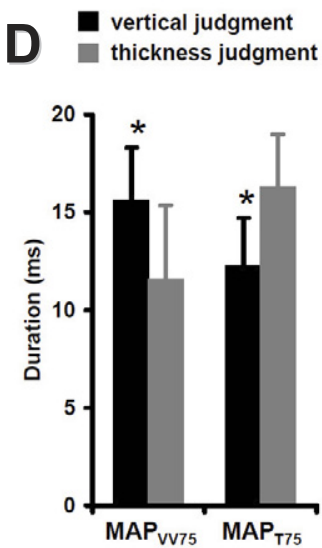

E $\square$ vertical judgment

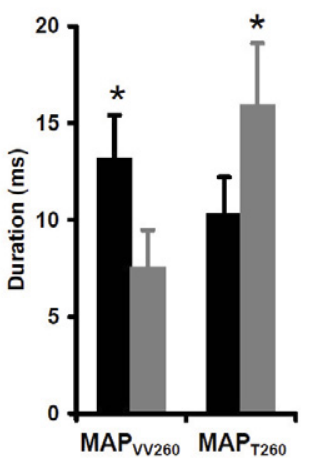

Fig. 5. Results from the topographic EP analysis. Segments of stable map topography for each visual task performed without visual reference (A) and with a tilted frame (B) are displayed under the global field power curve from 0 to $400 \mathrm{~ms}$ post-stimulus. Brain activity represented by EP maps at $\sim 75-105$ and at $\sim 260-290$ ms differed between visual vertical judgments (Map $\mathrm{V}_{\mathrm{V} 75}$ and $\mathrm{Map}_{\mathrm{VV} 260}$ ) and thickness judgments (Map $\mathrm{T}_{\mathrm{T} 55}$ and Map $_{\mathrm{T} 260}$ ). (C) Illustration of scalp topography corresponding to the EP maps found for the early and late processing stages (top view of a spherical representation of the scalp with the nasion on the top and the left scalp on the left). (D) Results of the competitive fitting of Map viv75 vs. Map $_{775}$ for the vertical and thickness judgments across participants. The duration of $M_{a v} p_{v 75}$ was significantly longer during vertical than thickness judgments, whereas the

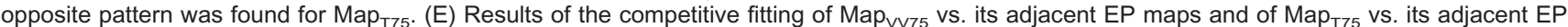
maps as a function of the visual reference. (F) Results of the competitive fitting of Map $\mathrm{vV} 260 \mathrm{vs}$ Map $_{\mathrm{T} 260}$ for the vertical and thickness judgments across participants. The duration of Map $\mathrm{v}_{\mathrm{v} 260}$ was significantly longer during vertical than thickness judgments, whereas the opposite pattern was found for $\mathrm{Map}_{\mathrm{T} 260}$. Vertical bars represent the standard error of the mean. Statistical significant difference between conditions: ${ }^{*} P<0.05$. For interpretation of the references to color in this figure legend, the reader is referred to the Web version of this article.

neuroimaging studies using $\mathrm{fMRI}$ and PET are hampered by the fact that the supine position in the scanner interferes with V judgments. The present behavioral analysis revealed that the perceived $\mathrm{V}$ was accurate without disturbing visual references in the periphery, suggesting that participants relied on an accurate internal model of the vertical while performing 


\section{Source localization early brain activities}

A

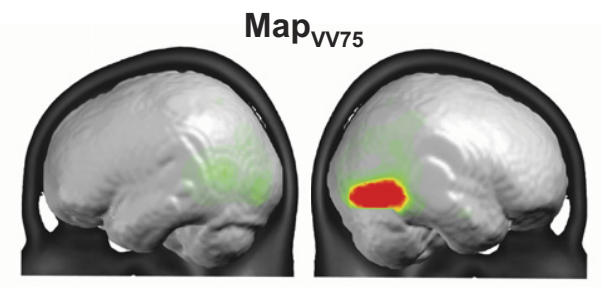

B

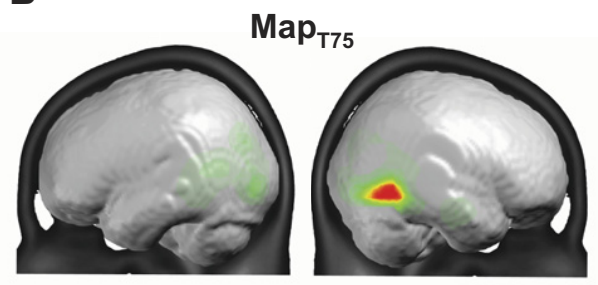

C

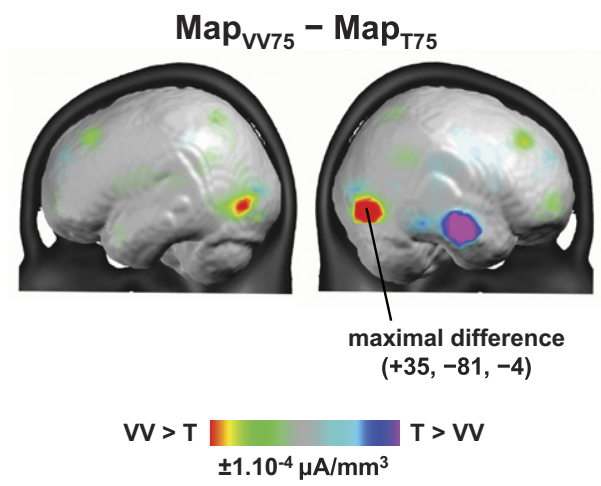

Source localization late brain activities

D

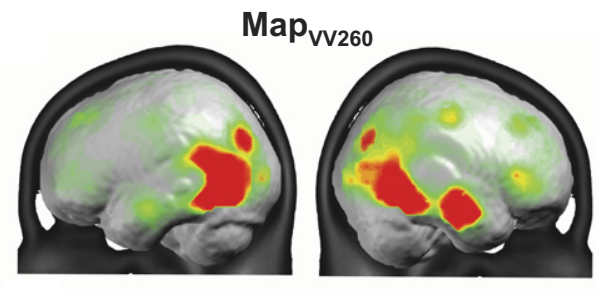

$5.10^{-4} \mu \mathrm{A} / \mathrm{mm}^{3}$

E

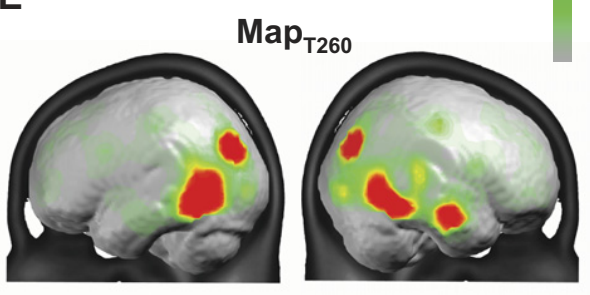

F

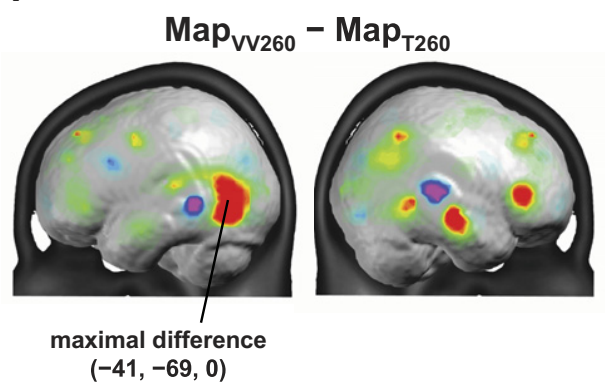

$\mathrm{VV}>\mathrm{T} \underset{ \pm 2.10^{-4} \mu \mathrm{A} / \mathrm{mm}^{3}}{\mathrm{~T}}>\mathrm{VV}$

Fig. 6. Group-averaged source estimations are shown for the early EP maps (A, B) and late EP maps (D, E) revealed by the segmentation procedure. Generators of EP Map $\mathrm{Vv}_{75}$ were found in the right temporo-occipital cortex, whereas generators of EP Map $\mathrm{vv}_{260}$ were localized to the left and right temporo-occipital cortex and left parieto-occipital cortex. (C, F) Mean differences between the sources estimations. For interpretation of the references to color in this figure legend, the reader is referred to the Web version of this article.

the $\mathrm{V}$ judgements. Our behavioral data also revealed that the $\mathrm{V}$ was significantly deviated towards the tilted visual references in the RFT, thus confirming previous observations in healthy populations (Guerraz et al., 1998; Lopez et al., 2006; Marendaz, 1998; Witkin and Asch, 1948; Zoccolotti et al., 1992). These effects were absent in the control task. Although the effect of the tilted frame was rather weak in the present study, this observation is in line with a recent report showing that 2D computer adaptations of the RFT evoked smaller V deviations than classical 3D RFT (Isableu et al., 2008). In addition, the mode of presentation of the visual lines (briefly flashed for $200 \mathrm{~ms}$ based on the constraints and the purpose of the present EP experiment) differs from that used in classical RFTs, where the rod that participants have to set vertical is continuously presented.

Regarding the present electrophysiological data, EP mapping indicated that $\mathrm{V}$ judgments were characterized by an early temporo-occipital activation, probably recruiting ex- trastriate cortex in the ventral stream, at $\sim 75-105 \mathrm{~ms}$ $\left(M_{a p}{ }_{v 75}\right)$. This was followed by a more distributed temporal, occipital, and parietal activation at $\sim 260-290 \mathrm{~ms}$ (Map ${ }_{\text {V260 }}$ ). These electrical neuroimaging data show that brain activation reflecting $\mathrm{V}$ judgments is distinct from brain activation during a control task that was matched for difficulty (as shown by similar reaction times) during two separate periods. In the following sections, we discuss these early and late brain mechanisms with respect to previous neuroimaging, neurophysiological, and lesion studies.

\section{Early activity in temporo-occipital cortex related to visual vertical judgments}

VV judgments led to a specific brain state at $\sim 75-105 \mathrm{~ms}$ (Map $_{V \text { 755 }}$ ) post-stimulus, revealing an influence of such judgments on early visual processing. This finding is supported by a significant difference in the amplitude of the 
A
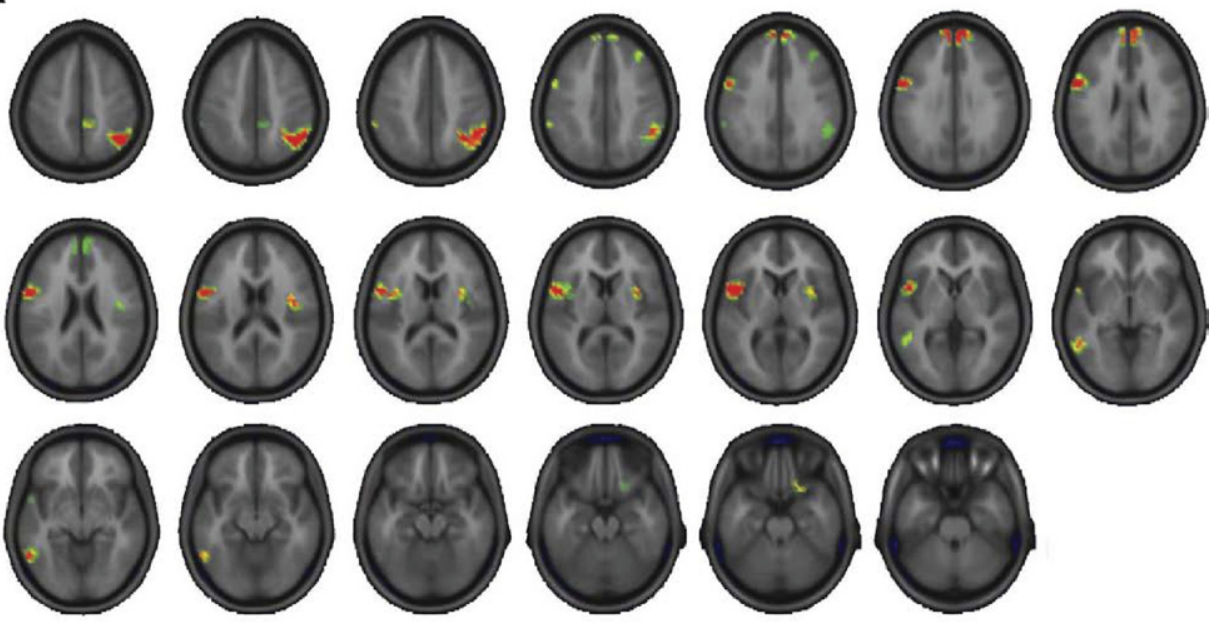

B
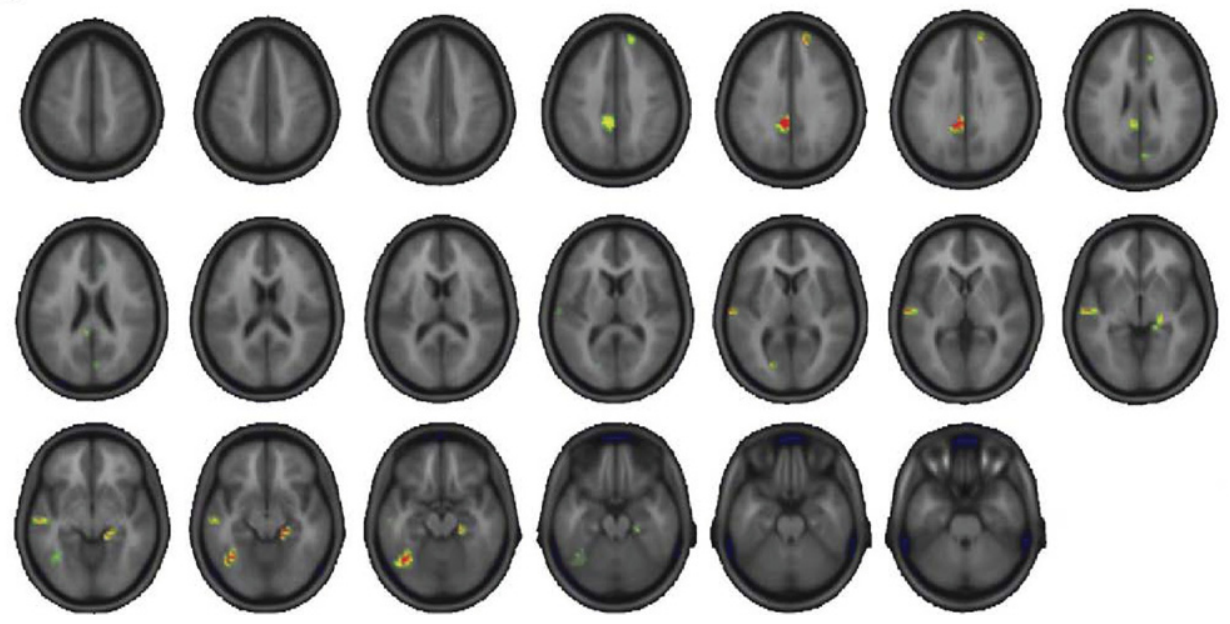

Fig. 7. Statistical analysis of the inverse solutions is shown for the comparison of the visual vertical judgments vs. the thickness judgments for the early brain activity (A: 75-105 ms, depicted at a significance level of $P<0.05$ uncorrected) and the later brain activity (B: $260-290 \mathrm{~ms}, P<0.01$ uncorrected). For interpretation of the references to color in this figure legend, the reader is referred to the Web version of this article.

GFP associated with VV and thickness judgments without visual references during the same time period. Early visual EP components at 50-90 ms (C1) and 80-130 ms (P1) after the presentation of visual stimuli have been shown to depend on visual stimulus characteristics such as luminance, contrast, color, form, motion, and orientation, as well as on stimulus position in the visual field (Celesia and Peachey, 2005; Clarck et al., 1995; Foxe and Simpson, 2002). The present early brain activity related to VV judgments overlapped mostly with the latency of the P1 component $(\sim 80-130 \mathrm{~ms})$ that has been related to the processing of, for example, luminance, contrast, position, and motion of visual stimuli (Celesia and Peachey, 2005; Itier and Taylor, 2004; Kuba et al., 2007). As contrast, luminance, form, and color were absolutely identical between $\mathrm{VV}$ and thickness judgment tasks, and because both tasks were characterized by the same reaction times, we argue that the observed electrophysiological dissociation between $\mathrm{V}$ and control task relates to task-relevant effects in temporo-occipital cortex that relate to orientation, comparison, or attention processes.

Our data suggest that brain activity around $100 \mathrm{~ms}$ may be important for specifically extracting information about visual orientation and/or for directing attention to visual orientation features. Recent electrophysiological investigations demonstrated that neural activity in striate and extrastriate cortical areas is modified by visual spatial attention occurring as early as $70-75 \mathrm{~ms}$ post-stimulus (Foxe and Simpson, 2002; Hillyard and AnlloVento, 1998; Martinez et al., 1999; Rauss et al., 2009). Consistent with our observation, Proverbio et al. (2002) found that visual attention to oriented gratings modulated brain activity at an early processing stage, around 80-140 ms. These authors suggested that the influence of the attentional selection of orientation on the $\mathrm{P} 1$ component, that was recorded at temporal electrodes, may reflect early brain mechanisms in the ventral visual stream. In the present experiment, it is difficult to ascer- 
tain how much attention to orientation features, vs. upright perception per se, was reflected in the activity pattern. Attention to orientation features was an important part of the task as participants were instructed to focus on this aspect of the visual stimuli (by opposition to paying attention to the thickness of the lines in the control task). However, the behavioral data show that the response times did not differ between both tasks, and did not differ as a function of the visual reference. This suggests that attention allocated to the visual stimuli was rather constant among tasks and visual stimuli. However, we cannot exclude that the attention to orientation features involved, or enhanced, specific neural mechanisms that were not present when paying attention to the thickness of the same lines. Here, we used a block design and asked participants to judge the orientation of visual lines with respect to gravity, repeatedly, with a large number of trials. Because participants implicitly had to focus on the orientation of the visual lines with respect to gravity, this may have modified the neural responses in orientation-sensitive regions in visual brain regions, whereas focused attention on the thickness of the same lines evoked a different brain activity at the same latency. Electrophysiological recordings in monkeys have indeed demonstrated an effect of attention on the neural mechanisms of orientation perception. When monkeys attended to visual stimuli, the responses of V4 neurons to oriented visual stimuli increased by $26 \%$, and those of V1 neurons increased by $8 \%$ (McAdams and Maunsell, 1999).

In addition to attention to orientation, the early brain mechanism may be indicative of processing of oriented visual stimuli in the ventral visual stream. The linear inverse solution localized $\mathrm{Map}_{\mathrm{V} v 75}$ in the ventral visual stream at the level of the right temporo-occipital cortex. The implication of the lateral occipital cortex has been shown in many previous neuroimaging studies dealing for example, with the perception of object shape (Kourtzi and Kanwisher, 2001) and object recognition (GrillSpector et al., 1999). Early VV brain activity is also close to regions at the right temporo-occipital cortex involved in the discrimination of the orientation of $2 \mathrm{D}$ visual objects (Faillenot et al., 1999). The temporal-occipital cortex has also been found to be involved in the discrimination or detection of the orientation of gratings presented successively (Altmann et al., 2005; Faillenot et al., 2001; Fias et al., 2002; Orban et al., 1997; Orban and Vogels, 1998) or simultaneously (Dupont et al., 1998). Accordingly, the early brain activation may reflect specialized processing related to orientation per se. Finally, although the contribution of extraretinal signals to ventral stream processing is weaker and has been less often reported than to dorsal stream processing (e.g. Tomko et al., 1981), we cannot clearly establish whether extraretinal signals such as gravitational vestibular cues, proprioceptive, and visceral interoceptive cues reflect the early ventral brain activity mechanism.
Late activity in temporo-occipital and parietooccipital cortex related to visual vertical judgments

EP mapping revealed the presence of a second brain activation related to $\mathrm{VV}$ judgments $\left(\mathrm{Map}_{\mathrm{Vv} 260}\right)$ appearing between $\sim 260$ and $290 \mathrm{~ms}$ after the presentation of the visual line segments. At the scalp level Map ${ }_{v v 260}$ was strongest at parietal electrodes. It had a latency close to the P3 component that has been related to high-level cognitive processing (Picton, 1992). In an EEG investigation of the neural basis of line-bisection judgments, Foxe et al. (2003) found a brain activation from $\sim 170 \mathrm{~ms}$ to $400 \mathrm{~ms}$ post-stimulus that was related to object-centered judgments and recorded at parietal and parieto-occipital scalp regions. In addition, an influence of attention on orientation feature detection has been shown around 270-430 ms after the presentation of oriented gratings, revealing a latter attentional influence than that described on P1 (Proverbio et al., 2002). In a recent investigation of line orientation perception with an adaptation of the RFT, the amplitude of P3 (but not of P1 and N1) was modulated by the orientation of a square frame surrounding a visual line (Corbett et al., 2009). In that latter study, the amplitude of P3 was larger when the orientation of the frame was incongruent to that of the tilted line segment as compared to when it was in a congruent orientation, suggesting that visual signals influence $\mathrm{VV}$ judgments at a late stage of visuo-spatial processing. However, a confounding factor in this experiment was that performances differed between the congruent and incongruent trials, because participants responded faster to incongruent trials.

Our data revealed neural differences underlying the processing of orientation of visual stimuli and of the thickness of two visual lines. We note that only few studies described brain mechanisms around this latency that are involved in orientation perception. Heinrich et al. (2008) found that the amplitude of P3 at the level of the parietal electrodes was modulated by the orientation of a grid composed of oriented elements continuously in movement (with larger responses for oblique orientations of the grid). The application of a linear inverse solution to Map $v$ v260 showed that this later mechanism for $\mathrm{VV}$ judgments involves a bilateral posterior network that was, in addition to the earlier brain activity, located more dorsally and also included parieto-occipital and temporal regions. This extends previous brain imaging findings demonstrating that orientation detection (of two visual stimuli presented successively) involves the left occipital gyrus, the right inferior temporal gyrus, and the left intraparietal sulcus (Fias et al., 2002). Fias and colleagues (2002) stressed that both ventral and dorsal streams are involved in orientation discrimination tasks, but that the detection of a difference (same/ different orientations) may involve more the ventral stream, while the quantification of the difference between the orientations of two visual stimuli may involve more strongly the dorsal stream. It is important to note that previous neuroimaging studies differ with respect to our study because they usually involved the judgment of the orientation of two visual stimuli successively presented (Orban and 
Vogels, 1998), while in our study the participants had to judge the orientation of visual stimuli with respect to their internal representation of the vertical, a canonical orientation not visually presented, but perceived through several sensory systems. The implication of the ventral stream in orientation discrimination is supported by electrophysiological recordings in monkeys showing that neurons in the inferior temporal cortex respond to orientation discrimination of successively presented gratings (Vogels and Orban, 1994). Several neuroimaging studies revealed the contribution of the ventral temporo-occipital regions to the discrimination of visual stimuli such as gratings (Dupont et al., 1998; Faillenot et al., 1999, 2001; Orban et al., 1997), simple 2D shapes (Faillenot et al., 1999), and pictures of objects (Altmann et al., 2005). The present implication of the dorsal stream extends previously reported dorsal stream contributions to orientation discrimination of visual stimuli (Faillenot et al., 1999, 2001; Fias et al., 2002) and hands (Taira et al., 1998). Our data are also in partial agreement with observations in brain-damaged patients as deviations of the $\mathrm{V} V$ have been reported after lesion of the temporo-parietal junction, including the superior temporal gyrus and inferior parietal lobule. Previous clinical studies have emphasized the importance of the insula (Brandt et al., 1994; Barra et al., 2010) and the posterior parietal cortex (Darling et al., 2003; Pérennou et al., 2008) in sensing gravity, and perceiving the $\mathrm{VV}$. The source localization applied to $\mathrm{MAP}_{\mathrm{VV} 260}$ did not reveal activation of the insula, but rather of more posterior and ventral regions. Detecting signals from the insula may be an inherent limitation of the EEG method because deep sources may be weak and underestimated by the inverse solution model (Michel et al., 2004). However, statistics on the inverse solution are indicative of a contribution of the insula, although not significant after Bonferroni correction (see Fig. 7). We believe that these differences may be partly due to the nature of the task involved. Although classical VV judgments, as routinely performed in clinics, involve that patients actively align a rod or laser to the perceived vertical, this was not the case in the present study. In our experimental conditions (chosen for the EP experiment), the task was purely visual and it did not involve repetitive motor commands for the readjustments of the visual stimuli orientation, apart from a single key press to validate the participant's answer.

As noted for the early brain activity underlying $\mathrm{VV}$ judgments, later brain activity represented by Map $\mathrm{V}_{\mathrm{V} 260}$ may be related to integration of extraretinal signals used for comparing the orientation of the segments of lines with gravity and one's body axis. The latency of Map $\mathrm{vv}_{\mathrm{V} 260}$ found in our study is compatible with that of previously described EP maps involved in perspective taking, or simulation of the body orientation (when participants mentally change their position in space with respect to gravity; Blanke et al., 2005; Tadi et al., 2009). This is of importance for the present study because $\mathrm{VV}$ judgments require that the brain detects the visual line's orientation with respect to our body as well as the orientation of our body with respect to gravity (Lopez et al., 2008, 2009; Luyat et al., 1997;
Marendaz, 1998; Mittelstaedt, 1992). Neural processes at this time period have also been shown to depend on the degree of elevation of the visuo-spatial perspective with respect to gravity (Schwabe et al., 2009), or to the participant's own body position (Arzy et al., 2006). Altogether, these high-density EP data suggest that brain mechanisms around $300 \mathrm{~ms}$ may represent multisensory integration for object-centered processing as well as for computing the position of one's own body in space (e.g. through vestibular, visceral, and muscular proprioceptive signals) used for $\mathrm{V}$ judgments.

In conclusion, the present data indicate that the later activations during VV judgments involve temporo-occipital and parieto-occipital cortex. Both regions have been involved in orientation-dependent visual processing. We speculate that the dorsal activation represents the integration of multisensory signals that are necessary for the accurate representation of the vertical. This is also concordant with clinical data showing that damage to the parietal cortex induces multimodal deficits of orientation perception (Kerkhoff, 1999).

\section{Hemispheric dominance for visual vertical judgment}

Early and late activation patterns were characterized by bilateral activations (see Fig. 6), but predominated on one side: we found a right hemispheric dominance for visual vertical judgment for Map $\mathrm{vv75}_{\mathrm{V} 5}$ and a left dominance for Map $_{\mathrm{vv260}}$.

We note that comparable lateralization of early brain activation has previously been reported in EEG investigations. For example, Foxe et al. (2003) investigated the neural basis of line bisection judgments and showed that the first phase revealed by the topography mapping has generators located in the right temporo-parietal junction and right lateral occipital cortex. These authors thus suggested a right hemisphere control of visuospatial perception. Early visual ERP recorded during presentation of visual stimuli influencing both hemifields have also been showed to involve the lateral occipital cortex in a nonsymmetrical way (Shpaner et al., 2009). A dominance of the right cerebral hemisphere for the representation of the egocentric and allocentric frames of reference has also been reported in fMRI studies (Vallar et al., 1999; Galati et al., 2001). In addition, the right hemisphere is also dominant for the control of body stabilization (Pérennou et al., 1997).

The later brain activation was rather associated with bilateral activation, although a left predominance was observed. Previous fMRI studies on the judgment of the position of lines with respect to the body mid-sagittal plane have revealed bilateral cortical networks with a hemispheric dominance (Vallar et al., 1999; Galati et al., 2001; Fink et al., 2003). Interestingly, the activation of both cerebral hemispheres during $\mathrm{VV}$ judgments corroborates clinical data. Damage to the right as well as the left insula can impair VV perception (Brandt et al., 1994). Moreover, the amplitude of deviation of the $\mathrm{V} V$ did not differ between right and left brain-damaged patients (Pérennou et al., 2008; Barra et al., 2010). 
Finally, because the visual stimuli equally covered both visual hemifields, and because this was the case in the experimental conditions ( $\mathrm{V} V$ judgments) as well as the control conditions (thickness judgments), the lateralization of brain activity that we found cannot be attributed to a stronger stimulation of one or the other visual hemifields. The laterality revealed in the present study has therefore to be related to brain mechanisms underlying $\mathrm{V} V$ judgments.

\section{Visual references and visual vertical judgments}

Behavioral analysis revealed that the perceived $\mathrm{V} V$ was significantly deviated towards the tilted visual references, indicating that participants relied on available external, allocentric, references to perform vertical judgments (Bray et al., 2004; Isableu et al., 1997; Lopez et al., 2006; Luyat et al., 1997; Marendaz, 1998). However, the EP mapping did not reveal any significant EP map reflecting the influence of the visual reference on behavior. Competitive fitting of $M P_{V V 75}$ with its adjacent EP maps (Fig. 5E) revealed that this map was longer during $V V$ judgments without visual reference than with visual reference (by contrast, $\mathrm{MAP}_{\mathrm{T} 75}$, had the same duration irrespective of the visual reference). This is indicative of an early influence of the visual reference, compatible with the view that during orientation judgments, multisensory integration occurs at an early stage of sensory processing (Marendaz, 1998). Moreover, the analysis of the amplitude of the GFP revealed significant difference for $\mathrm{VV}$ judgments performed with and without visual references during a time period starting $260 \mathrm{~ms}$ after stimulus presentation (Fig. 4C). Such observation corroborates results from a previous electrophysiological study showing an influence of a tilted frame on VV judgments at $\sim 300$ ms post-stimulus (Corbett et al., 2009). The authors suggested an influence of tilted visual references on post-perceptual level of orientation processing. This study, however, did not compare VV judgments with and without visual references. In the present experiment, the visual information presented at the beginning of each trial was the same (two segments of lines briefly flashed) for both conditions with and without visual references, and only the visual context differed between conditions. This could partly explain why we observed no EP map related to the visual reference. Moreover, the fact that the frame effect was rather weak as compared to that reported with classical 3D RFTs (Isableu et al., 2008), and the fact that normally participants have to actively align a movable line to the vertical, may explain some behavioral divergence with previous studies as well as the absence of an EP map related to the influence of the tilted visual environment on $\mathrm{VV}$ judgments.

\section{CONCLUSIONS}

The present study provides the first description of the location and timing of brain activity of $\mathrm{VV}$ judgments in a healthy population. We found that such $\mathrm{V} V$ judgments involve temporo-occipital and parieto-occipital regions at one early and one later period. We speculate that the early ventral stream activation reflects visual mechanisms that may depend on attention to orientation and comparison of visual features in regions including populations of orientation-dependent neurons. The later ventral-dorsal stream activation may reflect-next to orientation-dependent mechanisms-multisensory integration in the temporo-occipital and parieto-occipital regions, in line with the necessity to take into account one's own body position in space and other environmental constraints to determine how visual lines are oriented with respect to the vertical. Altogether, these data suggest that the brain has developed neural mechanisms subtending a constantly available and updated internal model of the vertical that we can refer to for the control of one's erect posture and actions (Dyde et al., 2006; Lopez et al., 2009; Marendaz, 1998) and various types of visuo-spatial processing.

Acknowledgments-The Cartool software (http://brainmapping. unige.ch/Cartool.htm) has been programmed by Denis Brunet, from the Functional Brain Mapping Laboratory, Geneva, Switzerland, and is supported by the Center for Biomedical Imaging (CIBM)) of Geneva and Lausanne. Christophe Lopez and Olaf Blanke are supported by the Swiss National Science Foundation (grant number SINERGIA CRSII1-125135/1). Manuel Mercier received support from a postdoctoral fellowship awarded by the Swiss National Science Foundation (PBELP3-123067).

\section{REFERENCES}

Altmann CF, Grodd W, Kourtzi Z, Bulthoff HH, Karnath HO (2005) Similar cortical correlates underlie visual object identification and orientation judgment. Neuropsychologia 43:2101-2108.

Angelaki DE, Shaikh AG, Green AM, Dickman JD (2004) Neurons compute internal models of the physical laws of motion. Nature 430:560-564.

Appelle S (1972) Perception and discrimination as a function of stimulus orientation: the "oblique effect" in man and animals. Psychol Bull 78:266-278.

Arzy S, Thut G, Mohr C, Michel CM, Blanke O (2006) Neural basis of embodiment: distinct contributions of temporoparietal junction and extrastriate body area. J Neurosci 26:8074-8081.

Barra J, Marquer A, Joassin R, Raymond C, Chauvineau V, Metge L, Pérennou D (2010) Human use internal models to construct and update a sense of verticality. Brain 133:3552-3563.

Blanke O, Mohr C, Michel CM, Pascual-Leone A, Brugger P, Seeck M, Landis T, Thut G (2005) Linking out-of-body experience and self processing to mental own-body imagery at the temporoparietal junction. J Neurosci 25:550-557.

Brandt T, Dieterich M, Danek A (1994) Vestibular cortex lesions affect the perception of verticality. Ann Neurol 35:403-412.

Bray A, Subanandan A, Isableu B, OhImann T, Golding JF, Gresty MA (2004) We are most aware of our place in the world when about to fall. Curr Biol 14:R609-R610.

Bronstein AM (1999) The interaction of otolith and proprioceptive information in the perception of verticality. The effects of labyrinthine and CNS disease. Ann N Y Acad Sci 871:324-333.

Celesia GG, Peachey NS (2005) Visual evoked potentials and electroretinograms. In: Electroencephalography. Basic principles, clinical applications, and related fields, fifth edition (Niedermeyer E, Lopes Da Silva F, eds), pp 1017-1043. Philadelphia: Lippincott Williams \& Wilkins.

Clarck VP, Fan S, Hillyard SA (1995) Identification of early visual evoked potential generators by retinotopic and topographic analyses. Hum Brain Mapp 2:170-187. 
Coppola DM, White LE, Fitzpatrick D, Purves D (1998) Unequal representation of cardinal and oblique contours in ferret visual cortex. Proc Natl Acad Sci U S A 95:2621-2623.

Corbett JE, Enns JT, Handy TC (2009) Electrophysiological evidence for a post-perceptual influence of global visual context on perceived orientation. Brain Res 1292:82-92.

Darling WG, Pizzimenti MA, Rizzo M (2003) Unilateral posterior parietal lobe lesions affect representation of visual space. Vision Res 43:1675-1688.

Dichgans J, Held R, Young LR, Brandt T (1972) Moving visual scenes influence the apparent direction of gravity. Science 178:1217-1219.

Dupont P, Vogels R, Vandenberghe R, Rosier A, Cornette L, Bormans G, Mortelmans L, Orban GA (1998) Regions in the human brain activated by simultaneous orientation discrimination: a study with positron emission tomography. Eur J Neurosci 10:3689-3699.

Dyde RT, Jenkin MR, Harris LR (2006) The subjective visual vertical and the perceptual upright. Exp Brain Res 173:612-622.

Faillenot I, Decety J, Jeannerod M (1999) Human brain activity related to the perception of spatial features of objects. Neuroimage 10:114-124.

Faillenot I, Sunaert S, Van Hecke P, Orban GA (2001) Orientation discrimination of objects and gratings compared: an fMRI study. Eur J Neurosci 13:585-596.

Fias W, Dupont P, Reynvoet B, Orban GA (2002) The quantitative nature of a visual task differentiates between ventral and dorsal stream. J Cogn Neurosci 14:646-658.

Fink GR, Marshall JC, Weiss PH, Stephan T, Grefkes C, Shah NJ, Zilles K, Dieterich M (2003) Performing allocentric visuospatial judgments with induced distortion of the egocentric reference frame: an fMRI study with clinical implications. Neuroimage 20:1505-1517.

Foxe JJ, McCourt ME, Javitt DC (2003) Right hemisphere control of visuospatial attention: line-bisection judgments evaluated with high-density electrical mapping and source analysis. Neuroimage 19:710-726.

Foxe JJ, Simpson GV (2002) Flow of activation from V1 to frontal cortex in humans. A framework for defining "early" visual processing. Exp Brain Res 142:139-150.

Furmanski CS, Engel SA (2000) An oblique effect in human primary visual cortex. Nat Neurosci 3:535-536.

Galati G, Committeri G, Sanes JN, Pizzamiglio L (2001) Spatial coding of visual and somatic sensory information in body-centred coordinates. Eur J Neurosci 14:737-746.

Gonzalez Andino SL, Grave de Peralta Menendez R, Lantz CM, Blank O, Michel CM, Landis T (2001) Non-stationary distributed source approximation: an alternative to improve localization procedures. Hum Brain Mapp 14:81-95.

Grave de Peralta Menendez R, Murray MM, Michel CM, Martuzzi R, Gonzalez Andino SL (2004) Electrical neuroimaging based on biophysical constraints. Neuroimage 21:527-539.

Grill-Spector K, Kushnir T, Edelman S, Avidan G, Itzchak Y, Malach R (1999) Differential processing of objects under various viewing conditions in the human lateral occipital complex. Neuron 24:187-203.

Guerraz M, Poquin D, Ohlmann T (1998) The role of head-centric spatial reference with a static and kinetic visual disturbance. Percept Psychophys 60:287-295.

Hegemann S, Fitzek S, Fitzek C, Fetter M (2004) Cortical vestibular representation in the superior temporal gyrus. J Vestib Res 14:33-35.

Heinrich SP, Aertsen A, Bach M (2008) Oblique effects beyond lowlevel visual processing. Vision Res 48:809-818.

Hillyard SA, Anllo-Vento L (1998) Event-related brain potentials in the study of visual selective attention. Proc Natl Acad Sci U S A 95:781-787.

Indovina I, Maffei V, Bosco G, Zago M, Macaluso E, Lacquaniti F (2005) Representation of visual gravitational motion in the human vestibular cortex. Science 308:416-419.
Isableu B, Gueguen M, Fourre B, Giraudet G, Amorim MA (2008) Assessment of visual field dependence: comparison between the mechanical 3D rod-and-frame test developed by Oltman in 1968 with a 2D computer-based version. J Vestib Res 18:239-247.

Isableu B, OhImann T, Cremieux J, Amblard B (1997) Selection of spatial frame of reference and postural control variability. Exp Brain Res 114:584-589.

Itier RJ, Taylor MJ (2004) Effects of repetition learning on upright, inverted and contrast-reversed face processing using ERPs. Neuroimage 21:1518-1532.

Kaptein RG, Van Gisbergen JA (2004) Interpretation of a discontinuity in the sense of verticality at large body tilt. J Neurophysiol 91:2205-2214.

Kerkhoff G (1999) Multimodal spatial orientation deficits in left-sided visual neglect. Neuropsychologia 37:1387-1405.

Kourtzi Z, Kanwisher N (2001) Representation of perceived object shape by the human lateral occipital complex. Science 293: 1506-1509.

Kuba M, Kubova Z, Kremlacek J, Langrova J (2007) Motion-onset VEPs: characteristics, methods, and diagnostic use. Vision Res 47:189-202.

Lehmann D, Ozaki H, Pal I (1987) EEG alpha map series: brain micro-states by space-oriented adaptive segmentation. Electroencephalogr Clin Neurophysiol 67:271-288.

Lehmann D, Skrandies W (1980) Reference-free identification of components of checkerboard-evoked multichannel potential fields. Electroencephalogr Clin Neurophysiol 48:609-621.

Li B, Peterson MR, Freeman RD (2003) Oblique effect: a neural basis in the visual cortex. J Neurophysiol 90:204-217.

Lopez C, Bachofner C, Mercier M, Blanke O (2009) Gravity and observer's body orientation influence the visual perception of human body postures. J Vis 9:1.1-14.

Lopez C, Lacour M, Ahmadi AE, Magnan J, Borel L (2007) Changes of visual vertical perception: a long-term sign of unilateral and bilateral vestibular loss. Neuropsychologia 45:2025-2037.

Lopez C, Lacour M, Leonard J, Magnan J, Borel L (2008) How body position changes visual vertical perception after unilateral vestibular loss. Neuropsychologia 46:2435-2440.

Lopez C, Lacour M, Magnan J, Borel L (2006) Visual field dependence-independence before and after unilateral vestibular loss. Neuroreport 17:797-803.

Luyat M, Mobarek S, Leconte C, Gentaz E (2005) The plasticity of gravitational reference frame and the subjective vertical: peripheral visual information affects the oblique effect. Neurosci Lett 385(3):215-219.

Luyat M, Ohlmann T, Barraud PA (1997) Subjective vertical and postural activity. Acta Psychol (Amst) 95:181-193.

Maffei L, Campbell FW (1970) Neurophysiological localization of the vertical and horizontal visual coordinates in man. Science 167:386-387.

Mansfield RJ (1974) Neural basis of orientation perception in primate vision. Science 186:1133-1135.

Marendaz C (1998) Nature and dynamics of reference frames in visual search for orientation: implications for early visual processing. Psychol Sci 9:27-32.

Martinez A, Anllo-Vento L, Sereno MI, Frank LR, Buxton RB, Dubowitz DJ, Wong EC, Hinrichs H, Heinze HJ, Hillyard SA (1999) Involvement of striate and extrastriate visual cortical areas in spatial attention. Nat Neurosci 2:364-369.

McAdams CJ, Maunsell JH (1999) Effects of attention on orientationtuning functions of single neurons in macaque cortical area $\mathrm{V} 4$. J Neurosci 19:431-441.

Mercier M, Schwartz S, Michel CM, Blanke O (2009) Motion direction tuning in human visual cortex. Eur J Neurosci 29:424-434.

Merfeld DM, Zupan L, Peterka RJ (1999) Humans use internal models to estimate gravity and linear acceleration. Nature 398:615-618. 
Michel CM, Murray MM, Lantz G, Gonzalez S, Spinelli L, Grave de Peralta R (2004) EEG source imaging. Clin Neurophysiol 115:2195-2222.

Michel CM, Thut G, Morand S, Khateb A, Pegna AJ, Grave de Peralta R, Gonzalez S, Seeck M, Landis T (2001) Electric source imaging of human brain functions. Brain Res Brain Res Rev 36:108-118.

Mittelstaedt $\mathrm{H}$ (1983) A new solution to the problem of the subjective vertical. Naturwissenschaften 70:272-281.

Mittelstaedt H (1992) Somatic versus vestibular gravity reception in man. Ann N Y Acad Sci 656:124-139.

Morand S, Thut G, de Peralta RG, Clarke S, Khateb A, Landis T, Michel CM (2000) Electrophysiological evidence for fast visual processing through the human koniocellular pathway when stimuli move. Cereb Cortex 10:817-825.

Murray MM, Brunet D, Michel CM (2008a) Topographic ERP analyses: a step-by-step tutorial review. Brain Topogr 20:249-264.

Murray MM, Camen C, Spierer L, Clarke S (2008b) Plasticity in representations of environmental sounds revealed by electrical neuroimaging. Neuroimage 39:847-856.

Murray MM, Foxe DM, Javitt DC, Foxe JJ (2004) Setting boundaries: brain dynamics of modal and amodal illusory shape completion in humans. J Neurosci 24:6898-6903.

Oldfield RC (1971) The assessment and analysis of handedness: the Edinburgh inventory. Neuropsychologia 9:97-113.

Orban GA, Dupont P, Vogels R, Bormans G, Mortelmans L (1997) Human brain activity related to orientation discrimination tasks. Eur J Neurosci 9:246-259.

Orban GA, Vandenbussche E, Vogels R (1984) Human orientation discrimination tested with long stimuli. Vision Res 24:121-128.

Orban GA, Vogels R (1998) The neuronal machinery involved in successive orientation discrimination. Prog Neurobiol 55:117-147.

Pascual-Marqui RD, Michel CM, Lehmann D (1995) Segmentation of brain electrical activity into microstates: model estimation and validation. IEEE Trans Biomed Eng 42:658-665.

Pérennou DA, Amblard B, Laassel el M, Pélissier J (1997) Hemispheric asymmetry in the visual contribution to postural control in healthy adults. Neuroreport 8:3137-3141.

Pérennou DA, Mazibrada G, Chauvineau V, Greenwood R, Rothwell J, Gresty MA, Bronstein AM (2008) Lateropulsion, pushing and verticality perception in hemisphere stroke: a causal relationship? Brain 131:2401-2413.

Perrin F, Pernier J, Bertrand O, Giard MH, Echallier JF (1987) Mapping of scalp potentials by surface spline interpolation. Electroencephalogr Clin Neurophysiol 66:75-81.

Picton TW (1992) The P300 wave of the human event-related potential. J Clin Neurophysiol 9:456-479.

Proverbio AM, Esposito P, Zani A (2002) Early involvement of the temporal area in attentional selection of grating orientation: an ERP study. Brain Res Cogn Brain Res 13:139-151.

Rauss KS, Pourtois G, Vuilleumier P, Schwartz S (2009) Attentional load modifies early activity in human primary visual cortex. Hum Brain Mapp 30:1723-1733.

Riccio GE, Martin EJ, Stoffregen TA (1992) The role of balance dynamics in the active perception of orientation. J Exp Psychol Hum Percept Perform 18:624-644.

Schwabe L, Lenggenhager B, Blanke O (2009) The timing of temporoparietal and frontal activations during mental own body transformations from different visuospatial perspectives. Hum Brain Mapp 30:1801-1812.

Seber GAF, Wild CJ (2003) Nonlinear regression. Hoboken, NJ: Wiley-Interscience.

Shpaner M, Murray MM, Foxe JJ (2009) Early processing in the human lateral occipital complex is highly responsive to illusory contours but not to salient regions. Eur J Neurosci 30:2018-2028.
Snyder L (1999) This way up: illusions and internal models in the vestibular system. Nat Neurosci 2:396-398.

Spierer L, Tardif E, Sperdin H, Murray MM, Clarke S (2007) Learninginduced plasticity in auditory spatial representations revealed by electrical neuroimaging. J Neurosci 27:5474-5483.

Tadi T, Overney LS, Blanke O (2009) Three sequential brain activations encode mental transformations of upright and inverted human bodies: a high resolution evoked potential study. Neuroscience 159:1316-1325.

Taira M, Kawashima R, Inoue K, Fukuda H (1998) A PET study of axis orientation discrimination. Neuroreport 9:283-288.

Talairach J, Tournoux P (1988) Co-planar stereotaxic atlas of the human brain. New York: Thieme.

Thierry G, Pegna AJ, Dodds C, Roberts M, Basan S, Downing P (2006) An event-related potential component sensitive to images of the human body. Neuroimage 32:871-879.

Thirioux B, Mercier M, Jorland G, Berthoz A, Blanke O (2010) Mental imagery of self-location during spontaneous and active self-other interactions: an electrical neuroimaging Study. J Neurosci 30:7202-7214.

Tomko DL, Barbaro NM, Ali FN (1981) Effect of body tilt on receptive field orientation of simple visual cortical neurons in unanesthetized cats. Exp Brain Res 43:309-314.

Trousselard M, Barraud PA, Nougier V, Raphel C, Cian C (2004) Contribution of tactile and interoceptive cues to the perception of the direction of gravity. Brain Res Cogn Brain Res 20:355-362.

Vaitl D, Mittelstaedt H, Baisch F (1997) Shifts in blood volume alter the perception of posture. Int J Psychophysiol 27:99-105.

Vallar G, Lobel E, Galati G, Berthoz A, Pizzamiglio L, Le Bihan D (1999) A fronto-parietal system for computing the egocentric spatial frame of reference in humans. Exp Brain Res 124:281-286.

Van Beuzekom AD, Medendorp WP, Van Gisbergen JA (2001) The subjective vertical and the sense of self orientation during active body tilt. Vision Res 41:3229-3242.

Van Beuzekom AD, Van Gisbergen JA (2000) Properties of the internal representation of gravity inferred from spatial-direction and body-tilt estimates. J Neurophysiol 84:11-27.

Vandenberghe R, Dupont P, De Bruyn B, Bormans G, Michiels J, Mortelmans L, Orban GA (1996) The influence of stimulus location on the brain activation pattern in detection and orientation discrimination. A PET study of visual attention. Brain 119 (Pt 4): 1263-1276.

Vingerhoets RA, De Vrijer M, Van Gisbergen JA, Medendorp WP (2009) Fusion of visual and vestibular tilt cues in the perception of visual vertical. J Neurophysiol 101:1321-1333.

Vogels R, Orban GA (1994) Activity of inferior temporal neurons during orientation discrimination with successively presented gratings. J Neurophysiol 71:1428-1451.

Witkin HA, Asch SE (1948) Studies in space orientation. IV. Further experiments on perception of the upright with displaced visual fields. J Exp Psychol 38:762-782.

Xu X, Collins CE, Khaytin I, Kaas JH, Casagrande VA (2006) Unequal representation of cardinal vs. oblique orientations in the middle temporal visual area. Proc Natl Acad Sci U S A 103:17490-17495.

Yelnik AP, Lebreton FO, Bonan IV, Colle FM, Meurin FA, Guichard JP, Vicaut E (2002) Perception of verticality after recent cerebral hemispheric stroke. Stroke 33:2247-2253.

Zink R, Bucher SF, Weiss A, Brandt T, Dieterich M (1998) Effects of galvanic vestibular stimulation on otolithic and semicircular canal eye movements and perceived vertical. Electroencephalogr Clin Neurophysiol 107:200-205.

Zoccolotti P, Antonucci G, Goodenough DR, Pizzamiglio L, Spinelli D (1992) The role of frame size on vertical and horizontal observers in the rod-and-frame illusion. Acta Psychol (Amst) 79:171-187. 